\title{
Design, Synthesis and Antitubercular Activity of Novel Isoniazid-Cyclic-Amine-Azachalcones Hybrids
}

\author{
Jefferson R. S. Oliveira, ${ }^{a}$ Cristiane Y. K. Shiguemoto, ${ }^{a}$ Amarith R. das Neves, ${ }^{a}$ \\ Flora M. F. Moreira, ${ }^{b}$ Giovana B. Gomes, ${ }^{c}$ Renata T. Perdomo, ${ }^{c}$ Sandro L. Barbosa, ${ }^{d}$ \\ Palimécio G. Guerrero Jr., ${ }^{e}$ Júlio Croda ${ }^{b, f}$ and Adriano C. M. Baroni ${ }^{\circledR} *, a$ \\ ${ }^{a}$ Laboratório de Síntese e Química Medicinal (LASQUIM), Faculdade de Ciências Farmacêuticas, \\ Alimentos e Nutrição, Universidade Federal do Mato Grosso do Sul (UFMS), \\ 79070-900 Campo Grande-MS, Brazil \\ ${ }^{b}$ Laboratório de Pesquisa em Ciências da Saúde (LPCS), \\ Universidade Federal da Grande Dourados (UFGD), 79925-070 Dourados-MS, Brazil \\ ${ }^{c}$ Laboratório de Biologia Molecular e Culturas Celulares (BioMol), \\ Faculdade de Ciências Farmacêuticas, Alimentos e Nutrição, \\ Universidade Federal do Mato Grosso do Sul (UFMS), 79070-900 Campo Grande-MS, Brazil \\ ${ }^{d}$ Departamento de Farmácia, Universidade Federal dos Vales do Jequitinhonha e Mucuri (UFVJM), \\ R. da Glória, 187, 39100-000 Diamantina-MG, Brazil \\ ${ }^{e}$ Laboratório de Síntese Orgânica, Departamento de Química e Biologia (DAQBi), \\ Universidade Tecnológica Federal do Paraná (UTFPR), 81280-340 Curitiba-PR, Brazil
}

${ }^{f}$ Fundação Oswaldo Cruz (Fiocruz) Mato Grosso do Sul, 79074-460 Campo Grande-MS, Brazil

\begin{abstract}
In this work, it is described the design of twenty-four heterocyclic amine-azachalcones compounds through molecular hybridization of chalcone scaffold and fragments of isoniazid, fluoroquinolones, and linezolid with antituberculosis potential. The new compounds were synthesized via Claisen-Schmidt condensation, providing yields of 36-95\%. Fifteen compounds showed antituberculosis activity against Mycobacterium tuberculosis $\mathrm{H} 37 \mathrm{Rv}$ strain. Two amine-azachalcones $\mathbf{1 5}$ and $\mathbf{1 7}$ showed relevant biological activity with minimum inhibitory concentration (MIC) values of 6.62 and $4.85 \mu \mathrm{M}$, respectively. Compound 12 showed the best profile of antitubercular activity with $\mathrm{MIC}=9.54 \mu \mathrm{M}$ and selectivity index $(\mathrm{SI})=9.33$. It was found that morpholine group is important to increase potency of antimycobacterial activity but also to add some toxicity to the chalcone molecular framework. The results described herein would be a guide in the designing of novel and optimized antitubercular derivatives based on the chalcone scaffold.
\end{abstract}

Keywords: isoniazid, heterocyclic amines, molecular hybridization, antitubercular activity, aldol condensation

\section{Introduction}

Tuberculosis (TB) disease is classified as one of the top ten leading causes of death in the world, due to its aggressive infection promoted by a unique agent. Moreover, one-fourth of the global population was detected with latent tuberculosis, occurring without any disease symptoms. ${ }^{1}$ In 2017, ten million people manifested TB and the situation aggravated even more with 1.3 million deaths. ${ }^{1}$

*e-mail: adriano.baroni@ufms.br
The first-line treatment for TB involves the use of five different drugs such as isoniazid, pyrazinamide, streptomycin, ethambutol, and rifampicin, which can be used in association. ${ }^{2}$ When the first-line drugs cannot be used, it is recommended the second-line treatment with levofloxacin or moxifloxacin, bedaquiline, and linezolid. ${ }^{3}$ Failures in chemotherapy are related to several aspects such as high complexity (association of three or more drugs); long period of treatment (6 to 9 months or up to 24 months), ${ }^{3,4}$ countless adverse effects (nausea, vomiting, and epigastric pain), ${ }^{1,5}$ and bacterial resistance. 
Bacterial resistance is a cause of alarm for the health systems throughout the world. ${ }^{3,6}$ Bacterial strains of TB have been classified into two types, TB multidrug-resistant (resistance to the first-line drugs), and TB extremely resistant (resistance to first and second-line drugs). ${ }^{6}$ In this context, it is reinforced the relevance to search into new antituberculosis drug candidates. ${ }^{7}$

Natural products remain a fundamental source of inspiration in the discovery and development of new bioactive compounds. ${ }^{8}$ Through secondary metabolism, natural products are able to create several types of substances, with multiple biological activities. ${ }^{9}$

Among the secondary metabolites, our research group is especially interested in chalcone scaffold, as a synthetic target. Chalcones are considered a privileged structure and have been widely used as a template in medicinal chemistry studies. Due to its structural diversity, chalcones have shown great potential in the molecular modification process, leading to the synthesis of compounds containing different pharmacophore groups, which can enhance its pharmacological effect. ${ }^{10}$

Recent reports ${ }^{10}$ aimed at chalcones as compounds with a wide range of biological activities such as anticancer, cancer-preventative effects, anti-inflammatory, antibacterial, antidiabetic, antioxidant, antimicrobial, antiviral, antimalarial and neuroprotective effects. It is noteworthy that chalcone compounds are emerging as potential candidates for new antitubercular drugs (Figure 1). ${ }^{10-15}$

Structure-activity relationship (SAR) and quantitative structure-activity relationship (QSAR) information has demonstrated that the nitrofuran scaffold as a substituent in chalcones 1-2 improved its antitubercular activities. ${ }^{16,17}$ However, many studies showed other structural combinations, aiming for new antitubercular agents, as seen in chalcones $\mathbf{3 - 4},{ }^{18,19}$ by the use of creative and intuitive molecular hybridization approach (Figure 1). ${ }^{20}$
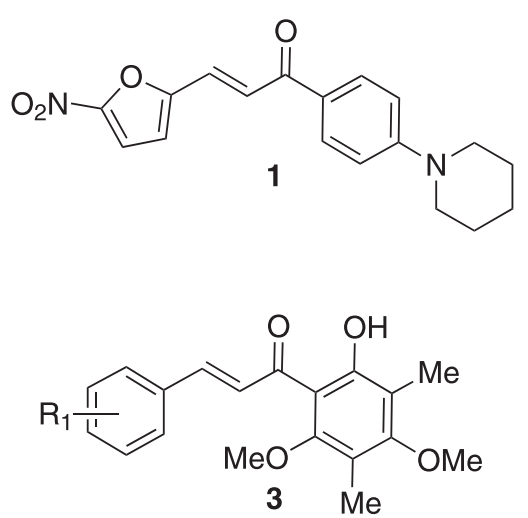

In order to expand the chemical space of chalcones, this study aimed to synthesize and evaluate the antitubercular activity of twenty-four heterocyclic amine-azachalcone derivatives designed by molecular hybridization of chalcone scaffold $\mathbf{5}$ with structural fragments of isoniazid $\mathbf{6}$, fluoroquinolones 7 and linezolid 8 (Scheme 1). Isoniazid 6 is the best and the oldest drug employed to treat TB, fluoroquinolone $\mathbf{7}$ and linezolid $\mathbf{8}$ are modern broadspectrum antibiotics, which have shown antitubercular activity. ${ }^{21-23}$

\section{Results and Discussion}

\section{Chemistry}

Firstly, it was necessary to obtain $p$-aminated acetophenones with the appropriate substitution patterns (Table 1). The synthesis of acetophenones 35a-35h occurred via aromatic nucleophilic substitution of 4-fluoroacetophenone 33 in the presence of cyclic amines 34a-34h, using $\mathrm{K}_{2} \mathrm{CO}_{3}$ and dimethyl sulfoxide (DMSO) as a solvent. Products were obtained in 71 to $99 \%$ yields; the reaction times were observed for 24 or $48 \mathrm{~h}$ until the complete consumption of starting materials (Scheme 2). ${ }^{24}$

Amine-azachalcones 9-32 were obtained via ClaisenSchmidt condensation of $p$-aminated acetophenones 35a-35h and carbaldehydes 36a-36c using a solution of $\mathrm{NaOH}$ in ethanol as solvent, which was added dropwise, providing the compounds 9-32 with yields ranging from 36 to $95 \%$ (Table 1 )..$^{25}$

Structural modifications intended to increase the complexity of cyclic amines bonded to the aromatic ring, in order to generate a variation in the molecular volume in R'. Thus, compounds were obtained from simple $N$-heterocyclic amine templates, as pyrrolidine, to more complex molecules like 1-(pyrimidin-2-yl)piperazine.

When positional isomers were compared, pyridine compounds substituted with nitrogen at position 4 showed<smiles>CSc1ccc(C(=O)/C=C/c2ccc([N+](=O)[O-])o2)cc1</smiles><smiles>COc1ccc(OC)c(C(=O)/C=C/c2ccc3nccnc3c2)c1</smiles>

Figure 1. Structures of new chalcone antitubercular agents 1-4. 
<smiles>O=C(/C=C/c1ccccc1)c1ccccc1</smiles>

5<smiles>NNC(=O)c1ccncc1</smiles>

Isoniazid 6<smiles>O=C(O)c1cn(C2CC2)c2cc(N3CCNCC3)c(F)cc2c1=O</smiles>

Fluoroquinolones 7<smiles>CC(=O)NCC1CN(c2ccc(N3CCOCC3)c(F)c2)C(=O)O1</smiles>

Molecular hybridization

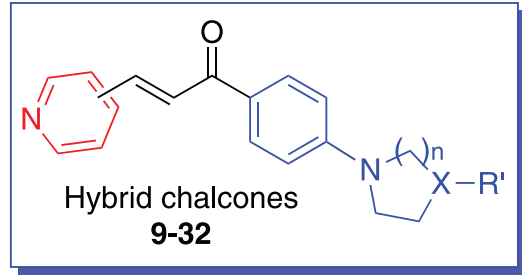

Scheme 1. Design of novel heterocyclic amines azachalcones analogues 9-32 by molecular hybridization of chalcone scaffold with fragments of isoniazid, fluoroquinolones and/or linezolid.

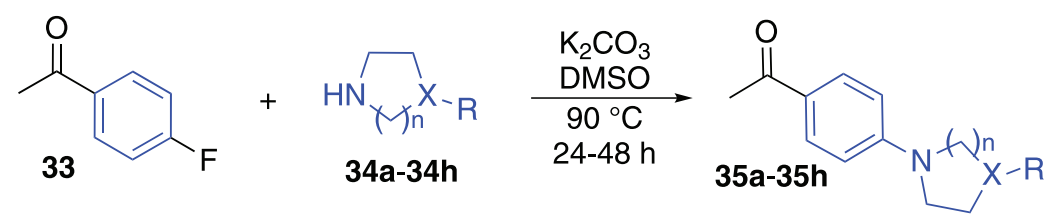

Scheme 2. Synthesis of $p$-aminated acetophenones 35a-35h. Reagents and reaction conditions: 33 (10 mmol), 34a-34h (17 $\mathrm{mmol}), \mathrm{K}_{2} \mathrm{CO}_{3}(17 \mathrm{mmol})$, $\operatorname{DMSO}(25 \mathrm{~mL}), 90^{\circ} \mathrm{C}, 24 \mathrm{~h}(\mathbf{3 5 a}-35 \mathrm{c}, 35 \mathrm{e}, 35 \mathrm{f})$ or $48 \mathrm{~h}(\mathbf{3 5 d}, \mathbf{3 5 g}, \mathbf{3 5 h})$.

the worst yields $(11,14,17,20,23,26,32)$ except for the compound 29.

Unknown synthetic compounds 9-32 were characterized by ${ }^{1} \mathrm{H}$ and ${ }^{13} \mathrm{C}$ nuclear magnetic resonance (NMR) and high-resolution mass spectrometry (HRMS).

\section{Antitubercular activity}

The antitubercular activity of compounds 9-32 was evaluated against Mycobacterium tuberculosis H37Rv strain (Table 2). Minimum inhibitory concentrations (MICs) were defined as the lowest concentration that resulted in $90 \%$ of M. tuberculosis growth inhibition. Fifteen compounds of the series demonstrated some level of activity against $M$. tuberculosis.

The presence of a six-membered hetero-aromatic ring bonding directly to the cyclic amine group did not contribute to the antituberculosis activity (MIC $>250 \mu \mathrm{M}$ ) since the bulkiest azachalcones 27-32 were inactive.

Hybrid pyrrolidine substituted derivatives 9 and 10 were also inactive against $M$. tuberculosis (MIC $>250 \mu \mathrm{M})$. However, compound 11 showed good activity (MIC $=20.22 \mu \mathrm{M}$ ).

Compounds 12-14, for instance, presented different biological activities. The isomers $\mathbf{1 2}$ and $\mathbf{1 4}$ exhibited values of $\mathrm{MIC}=9.54$ and $24.52 \mu \mathrm{M}$, respectively. On the other hand, isomer 13 was inactive (MIC $>250 \mu \mathrm{M}$ ).

Thiomorpholine derivatives 18-20 showed MIC values ranging from 34.79 to $111.13 \mu \mathrm{M}$. Bioisosteric substitution of sulfur atom (in thiomorpholine derivatives) by oxygen (morpholine nucleus) improved the biological activity. Compounds 15, 16 and 17 were considered the most active positional isomers in the series, exhibiting an MIC of 6.62, 14.03 , and $4.85 \mu \mathrm{M}$, respectively.

Additionally, $\mathrm{N}$-alkyl piperazine derivatives 21-23 ( $\mathrm{MIC}=14.15,26.64$ and $16.72 \mu \mathrm{M}$, respectively) displayed good biological activities when compared to pyrazinamide $(\mathrm{MIC}=26.80 \mu \mathrm{M})$.

Regarding SAR of positional isomers (2-pyridine, 3-pyridine, 4-pyridine), it was observed different MICs in the same structural group, indicating the importance of this factor in the biological activity. Derivatives containing 4-pyridines were more active than their 2- and 3- positional congeners (Table 2, compounds 11, 17, 20, 26). Positional isomers with 2-pyridine were the second most active (Table 2, compounds $\mathbf{1 2}$ and 21). Compounds with a 3-pyridine nucleus were the least active (Table 2, compounds 10, 13, 16, 22, 25).

In summary, the compounds with MIC $<10 \mu \mathrm{M}$ were considered good hits in a series of antituberculosis drug 
Vol. 31, No. 6, 2020

Oliveira et al.

1287

Table 1. Synthesis of the heterocyclic amine-azachalcones 9-32<smiles>[R5][Y]1([H])CCN(c2ccc(C(C)=O)cc2)C1</smiles><smiles>CCO[N+](=O)CCc1ccncc1</smiles><smiles>[R][Y]1([H])CCN(c2ccc(C(=O)/C=C/c3ccncc3)cc2)N1</smiles><smiles>O=C(/C=C/c1ccccn1)c1ccc(N2CCCC2)cc1</smiles><smiles>O=C(/C=C/c1cccnc1)c1ccc(N2CCCC2)cc1S(=O)(=O)[O-]</smiles><smiles>O=C(/C=C/c1ccncc1)c1ccc(N2CCCC2)cc1</smiles><smiles></smiles><smiles>O=C(/C=C/c1cccnc1)c1ccc(N2CCCCC2)cc1</smiles><smiles>O=C(/C=C/c1ccncc1)c1ccc(N2CCCCC2)cc1</smiles><smiles>O=C(/C=C/c1ccccn1)c1ccc(N2CCOCC2)cc1</smiles><smiles>O=C(/C=C/c1cccnc1)c1ccc(N2CCOCC2)cc1</smiles><smiles>O=C(/C=C/c1ccncc1)c1ccc(N2CCOCC2)cc1</smiles>

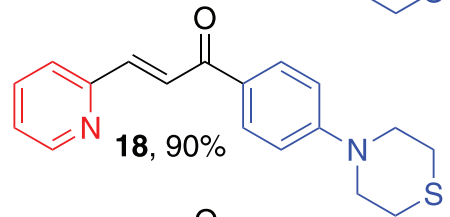<smiles>O=C(/C=C/c1cccnc1)c1ccc(N2CCSCC2)cc1[Hg]</smiles>

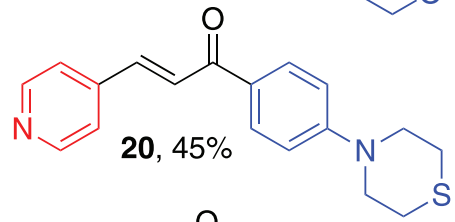<smiles>[Z10][R6]c1ccc(C(=O)/C=C/c2ccccn2)cc1</smiles>

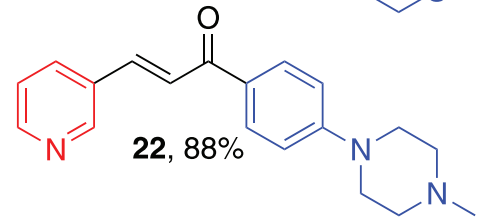

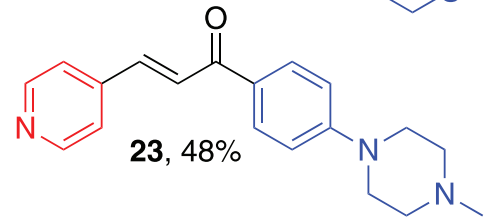<smiles>CCN1CCN(c2ccc(C(=O)/C=C/c3ccccn3)cc2)CC1</smiles><smiles>CCN1CCN(c2ccc(C(=O)/C=C/c3cccnc3)cc2)CC1</smiles><smiles>CCN1CCN(c2ccc(C(=O)/C=C/c3ccncc3)cc2)CC1</smiles>

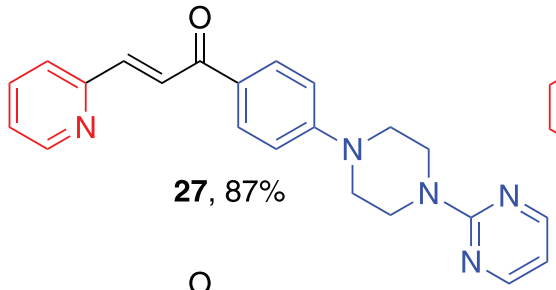

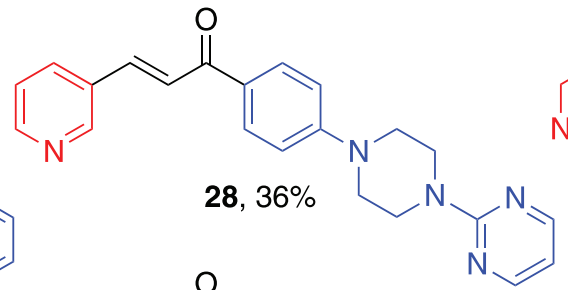<smiles>O=C(/C=C/c1ccncc1)c1ccc(N2CCN(c3ncccn3)CC2)cc1</smiles>

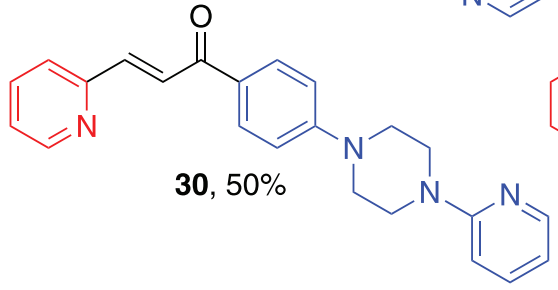<smiles>C/C=C\N</smiles><smiles>C/C=C\N</smiles>

Reaction conditions: 35a-35h ( $2 \mathrm{mmol})$, 36a-36c (4 mol), ethanol (10 mL) and $\mathrm{NaOH}$ (30 mol solubilize in $25 \mathrm{~mL}$ of ethanol added dropwise), room temperature, $4 \mathrm{~h}$, inert atmosphere. 
Table 2. In vitro antituberculosis activity of azachalcones 9-32 against M. tuberculosis H37Rv strain

\begin{tabular}{|c|c|c|c|c|}
\hline Compound & $\mathrm{H} 37 \mathrm{Rv} \mathrm{MIC}^{\mathrm{a}} /\left(\mu \mathrm{g} \mathrm{mL}^{-1}\right)$ & $\mathrm{H} 37 \mathrm{Rv} \mathrm{MIC}^{\mathrm{a}} / \mu \mathrm{M}$ & $\mathrm{NIH} / 3 \mathrm{~T} 3 \mathrm{IC}_{50}^{\mathrm{b}} /\left(\mu \mathrm{g} \mathrm{mL}^{-1}\right)$ & $\mathrm{SI}^{\mathrm{c}}$ \\
\hline 9 & $>250$ & $>250$ & 17.39 & 0.07 \\
\hline 10 & $>250$ & $>250$ & 32.21 & 0.13 \\
\hline 11 & 5.63 & 20.22 & 64.78 & 11.51 \\
\hline 12 & 2.79 & 9.54 & 26.04 & 9.33 \\
\hline 13 & $>250$ & $>250$ & 32.87 & 0.13 \\
\hline 14 & 7.17 & 24.52 & 2.87 & 0.40 \\
\hline 15 & 1.95 & 6.62 & 2.71 & 1.39 \\
\hline 16 & 4.13 & 14.03 & 2.94 & 0.71 \\
\hline 17 & 1.43 & 4.85 & 4.99 & 3.49 \\
\hline 18 & 34.50 & 111.13 & 79.16 & 2.29 \\
\hline 19 & 15.30 & 49.28 & 5.28 & 0.34 \\
\hline 20 & 10.80 & 34.79 & 2.78 & 0.26 \\
\hline 21 & 4.35 & 14.15 & 2.88 & 0.66 \\
\hline 22 & 8.19 & 26.64 & 20.73 & 2.53 \\
\hline 23 & 5.14 & 16.72 & 2.77 & 0.54 \\
\hline 24 & 3.62 & 11.26 & 2.71 & 0.75 \\
\hline 25 & 7.76 & 24.14 & 23.39 & 3.01 \\
\hline 26 & 3.38 & 10.51 & 2.60 & 0.77 \\
\hline 27 & $>250$ & $>250$ & 32.43 & 0.13 \\
\hline 28 & $>250$ & $>250$ & 361.58 & 1.44 \\
\hline 29 & $>250$ & $>250$ & 32.78 & 0.13 \\
\hline 30 & $>250$ & $>250$ & 23.21 & 0.09 \\
\hline 31 & $>250$ & $>250$ & 26.94 & 0.10 \\
\hline 32 & $>250$ & $>250$ & 29.42 & 0.11 \\
\hline $\mathrm{INH}^{\mathrm{d}}$ & 0.015 & 0.10 & $\mathrm{NC}^{\mathrm{e}}$ & $\mathrm{NC}^{\mathrm{e}}$ \\
\hline $\mathrm{RMP}^{\mathrm{f}}$ & 0.059 & 0.065 & $\mathrm{NC}^{\mathrm{e}}$ & $\mathrm{NC}^{\mathrm{e}}$ \\
\hline PYR $^{\mathrm{g}}$ & 3.3 & 26.80 & $\mathrm{NC}$ & $\mathrm{NC}^{\mathrm{e}}$ \\
\hline DOX $^{\mathrm{h}}$ & $\mathrm{NC}^{\mathrm{e}}$ & $\mathrm{NC}^{\mathrm{e}}$ & 2.50 & $\mathrm{NC}^{\mathrm{e}}$ \\
\hline
\end{tabular}

a MIC: minimum inhibitory concentration (lowest concentration that results in $90 \%$ inhibition of growth of $M$. tuberculosis); ${ }^{\mathrm{b} I \mathrm{C}_{50}}$ : half maximal inhibitory concentration on fibroblast cells (NIH/3T3); ' $\mathrm{SI}$ : selectivity index ( $\mathrm{IC}_{50}$ on fibroblast cells / MIC on M. tuberculosis H37Rv strain); ${ }^{\mathrm{d} I N H}$ : isoniazid, positive control for M. tuberculosis; ${ }^{\mathrm{N}} \mathrm{NC}$ : values not calculated; ${ }^{\mathrm{f}} \mathrm{RMP}$ : rifampicin, positive control for M. tuberculosis; ${ }^{\mathrm{PPYR}}$ : pyrazinamide, positive control for

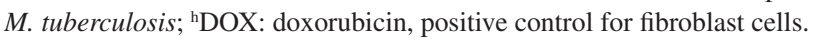

candidates. Azachalcones 12, 15 and $\mathbf{1 7}$ with $\mathrm{MIC}=9.54$ (selectivity index $(\mathrm{SI})=9.33), 6.62(\mathrm{SI}=1.39)$ and $4.85 \mu \mathrm{M}$ $(\mathrm{SI}=3.49)$ showed higher activity than the reference drug tested, pyrazinamide $(\mathrm{MIC}=26.80 \mu \mathrm{M})$. These derivatives also showed relevant antituberculosis profile when compared to ciprofloxacin $(\mathrm{MIC}=9.43 \mu \mathrm{M}) .{ }^{18}$ Figure 2 shows the SAR highlights of azachalcones.

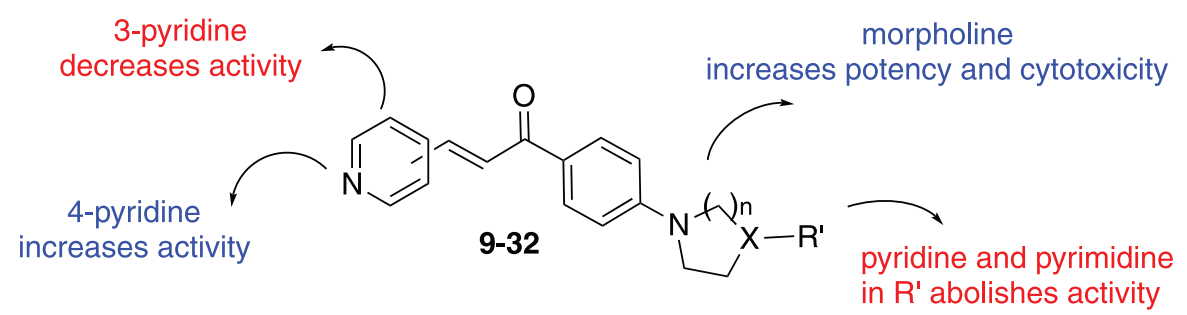

Figure 2. SAR of the hybrids azachalcones 9-32. 


\section{Conclusions}

In conclusion, twenty-four heterocyclic amineazachalcones 9-32 were obtained from moderate to good yields. Antituberculosis activity was evaluated against the $M$. tuberculosis $\mathrm{H} 37 \mathrm{Rv}$ strain. Although the higher potency of compounds $\mathbf{1 5}$ and $\mathbf{1 7}$ ( $\mathrm{MIC}=6.62$ and $4.85 \mu \mathrm{M}$, respectively) towards antituberculosis activity, both analogues were considered more cytotoxic (SI $=1.39$ and 3.49, respectively). However, azachalcone 12 showed the best profile of activity and selectivity index $(\mathrm{MIC}=9.54 \mu \mathrm{M} ; \mathrm{SI}=9.33)$, which provide important information about the SAR.

Morpholine group as a substituent in molecular template was related to higher potency and relevant biological activity, but on the other hand it also increased the cytotoxicity of azachalcones. Further research might explore new structural modifications, in order to provide more information about SAR of azachalcones, and also, optimize the hit-like compounds of this series.

\section{Experimental}

\section{General remarks}

All solvents were purchased from Synth ${ }^{\circledR}$, São Paulo, Brazil, and distilled before use according to the standard procedure. All reactions were performed under an atmosphere of dry nitrogen and monitored by thin-layer chromatography (TLC) using prepared plates (silica gel 60 F254 on aluminum purchased from SigmaAldrich $^{\circledR}$, St. Louis, MO, USA). The chromatograms were examined under both 254 and $360 \mathrm{~nm}$ UV light or with the developing agent ethanolic vanillin and heat. Flash column chromatography was performed on silica gel 60 (particle size 200-400 mesh ASTM, purchased from Sigma-Aldrich $^{\circledR}$, St. Louis, MO, USA) and eluted with hexane or hexane/ethyl acetate in different ratios. Melting points were determined using Fisatom 430D equipment. The ${ }^{13} \mathrm{C}$ and ${ }^{1} \mathrm{H}$ NMR spectra were recorded in $\mathrm{CDCl}_{3}$ solutions using a Bruker 75 or $300 \mathrm{MHz}$ spectrometer. Chemical shifts $(\delta)$ were expressed as parts per million (ppm) downfield from tetramethylsilane as the internal standard. High-resolution electrospray ionization mass spectrometry (HR-ESI-MS) measurements were carried out on a quadrupole time-of-flight instrument (UltrOTOF-Q, BrukerDaltonics, Billerica, MA, USA). General reagents as 4-fluoro-acetophenone 33, compound 35b, cyclic amines $\mathbf{3 4 a}, \mathbf{3 4 c - 3 4 h}$, carbaldehydes 36a-36c, bases, were purchased from Sigma-Aldrich ${ }^{\circledR}$, St. Louis, MO, USA.
General procedure for the preparation of $p$-aminated acetophenone (35a-35h)

To a solution of compounds 33 (10 $\mathrm{mmol})$, 34a-34h (17 mmol) in dimethyl sulfoxide $(25 \mathrm{~mL}), \mathrm{K}_{2} \mathrm{CO}_{3}$ (17 mmol) was added. The reaction was stirred at $90{ }^{\circ} \mathrm{C}$ for $24 \mathrm{~h}(\mathbf{3 5 a}, 35 \mathbf{c}, 35 \mathrm{e}, 35 \mathbf{f})$ or $48 \mathrm{~h} \mathrm{(35d,} \mathrm{35g,} \mathrm{35h).}$ After that, a saturated aqueous $\mathrm{NaCl}$ solution $(50 \mathrm{~mL})$ was added. Then, the reaction was extracted with ethyl acetate $(8 \times 25 \mathrm{~mL})$, the organic phase was washed with saturated aqueous $\mathrm{NaCl}$ solution $(10 \times 50 \mathrm{~mL})$. The aqueous phase was retro-extracted with ethyl acetate $(2 \times 25 \mathrm{~mL})$. Organic phases were dried over anhydrous $\mathrm{MgSO}_{4}$ and the solvent was removed under reduced pressure. Compounds 35a, 35c, 35e, $35 \mathbf{f}$ did not require further purification. Compounds 35d, 35g, 35h were purified by column chromatography on silica gel using hexane/ethyl acetate 8:2 as eluent.

\section{1-(4-(Pyrrolidin-1-yl)phenyl)ethanone (34a)}

Yield: 99\%; light brown solid; ${ }^{1} \mathrm{H}$ NMR (300 MHz, $\left.\mathrm{CDCl}_{3}\right) \delta 1.98-2.03\left(\mathrm{~m}, 4 \mathrm{H}, 2 \mathrm{CH}_{2}\right), 2.47\left(\mathrm{~s}, 3 \mathrm{H}, \mathrm{CH}_{3}\right), 3.33$ (m, 4H, 2CH $), 6.48$ (d, 2H, J $9.0 \mathrm{~Hz}, \mathrm{Ar}-\mathrm{H}), 7.83$ (d, 2H, $J 9.0 \mathrm{~Hz}, \mathrm{Ar}-\mathrm{H}) ;{ }^{13} \mathrm{C} \mathrm{NMR}\left(75 \mathrm{MHz}, \mathrm{CDCl}_{3}\right) \delta 25.4,25.9$, $29.7,110.6,124.9,130.7,150.9,196.3$.

\section{1-(4-Morpholinophenyl)ethanone (34c)}

Yield: 79\%; light brown solid; ${ }^{1} \mathrm{H}$ NMR (300 MHz, $\left.\mathrm{CDCl}_{3}\right) \delta 2.51\left(\mathrm{~s}, 3 \mathrm{H}, \mathrm{CH}_{3}\right), 3.27-3.32\left(\mathrm{~m}, 4 \mathrm{H}, 2 \mathrm{CH}_{2}\right)$, 3.81-3.86 (m, 4H, 2CH$), 6.79$ (d, 2H, J 9.0 Hz, Ar-H), 7.85 (d, 2H, J 9.0 Hz, Ar-H); ${ }^{13} \mathrm{C}$ NMR (75 MHz, $\mathrm{CDCl}_{3}$ ) $\delta$ 26.2, 47.5, 66.6, 113.3, 128.1, 130.3, 154.2, 196.4.

\section{1-(4-Thiomorpholinophenyl)ethanone (34d)}

Yield: 85\%; light yellow solid; ${ }^{1} \mathrm{H}$ NMR (300 MHz, $\left.\mathrm{CDCl}_{3}\right) \delta 2.49\left(\mathrm{~s}, 3 \mathrm{H}, \mathrm{CH}_{3}\right), 2.65-2.70\left(\mathrm{~m}, 4 \mathrm{H}, 2 \mathrm{CH}_{2}\right)$, 3.73-3.79 (m, 4H, 2CH $), 6.85$ (d, 2H, J 9.0 Hz, Ar-H), 7.88 (d, 2H, J $9.0 \mathrm{~Hz}, \mathrm{Ar}-\mathrm{H}) ;{ }^{13} \mathrm{C} \mathrm{NMR}\left(75 \mathrm{MHz}, \mathrm{CDCl}_{3}\right)$ $\delta 25.8,26.1,50.3,113.6,127.2,130.6,152.9,196.4$.

1-(4-(4-Methylpiperazin-1-yl)phenyl)ethanone (34e)

Yield: 90\%; light brown solid; ${ }^{1} \mathrm{H}$ NMR (300 MHz, $\left.\mathrm{CDCl}_{3}\right) \delta 2.38\left(\mathrm{~s}, 3 \mathrm{H}, \mathrm{CH}_{3}\right), 2.50\left(\mathrm{~s}, 3 \mathrm{H}, \mathrm{CH}_{3}\right), 2.58-2.63$ $\left(\mathrm{m}, 4 \mathrm{H}, 2 \mathrm{CH}_{2}\right), 3.37-3.42\left(\mathrm{~m}, 4 \mathrm{H}, 2 \mathrm{CH}_{2}\right), 6.86(\mathrm{~d}, 2 \mathrm{H}$, $J 9.0 \mathrm{~Hz}, \mathrm{Ar}-\mathrm{H}), 7.86$ (d, 2H, J 9.0 Hz, Ar-H); ${ }^{13} \mathrm{C}$ NMR $\left(75 \mathrm{MHz} \mathrm{CDCl}_{3}\right) \delta 26.1,45.9,47.1,54.6,113.5,127.8$, $130.4,153.9,196.5$.

\section{1-(4-(4-Ethylpiperazin-1-yl)phenyl)ethanone (34f)}

Yield: 94\%; beige solid; ${ }^{1} \mathrm{H}$ NMR $\left(300 \mathrm{MHz}, \mathrm{CDCl}_{3}\right)$ $\delta 1.09$ (t, 3H, J $6.0 \mathrm{~Hz}, \mathrm{CH}_{3}$ ), 2.43 (q, 2H, J 6.0 Hz, $\mathrm{CH}_{2}$ ), $2.48\left(\mathrm{~s}, 3 \mathrm{H}, \mathrm{CH}_{3}\right), 2.52-2.57\left(\mathrm{~m}, 4 \mathrm{H}, 2 \mathrm{CH}_{2}\right), 3.32-3.37$ 
$\left(\mathrm{m}, 4 \mathrm{H}, 2 \mathrm{CH}_{2}\right), 6.83(\mathrm{~d}, 2 \mathrm{H}, J 9.0 \mathrm{~Hz}, \mathrm{Ar}-\mathrm{H}), 7.84(\mathrm{~d}, 2 \mathrm{H}$, $J 9.0 \mathrm{~Hz}, \mathrm{Ar}-\mathrm{H}) ;{ }^{13} \mathrm{C} \mathrm{NMR}\left(75 \mathrm{MHz}, \mathrm{CDCl}_{3}\right) \delta 11.9,26.1$, $47.2,52.3,52.4,113.3,127.5,130.3,154.1,196.5$.

\section{1-(4-(4-(Pyrimidin-2-yl)piperazin-1-yl)phenyl)ethanone} (34g)

Yield: 92\%; light brown solid; ${ }^{1} \mathrm{H}$ NMR $(300 \mathrm{MHz}$, $\left.\mathrm{CDCl}_{3}\right) \delta 2.49\left(\mathrm{~s}, 3 \mathrm{H}, \mathrm{CH}_{3}\right), 3.39-3.44\left(\mathrm{~m}, 4 \mathrm{H}, 2 \mathrm{CH}_{2}\right)$, $3.95\left(\mathrm{~m}, 4 \mathrm{H}, 2 \mathrm{CH}_{2}\right), 6.50(\mathrm{t}, 1 \mathrm{H}, J 4.8 \mathrm{~Hz}, \mathrm{Ar}-\mathrm{H}), 6.87$ (d, 2H, J 9.0 Hz, Ar-H), 7.87 (d, $2 \mathrm{H}, J 9.0 \mathrm{~Hz}, \mathrm{Ar}-\mathrm{H})$, $8.31(\mathrm{~d}, 2 \mathrm{H}, J 4.8 \mathrm{~Hz}, \mathrm{Ar}-\mathrm{H}) ;{ }^{13} \mathrm{C} \mathrm{NMR}\left(75 \mathrm{MHz}, \mathrm{CDCl}_{3}\right)$ $\delta 26.2,43.3,47.2,110.3,113.5,127.8,130.4,154.0$, $157.8,161.4,196.5$.

\section{1-(4-(4-(Pyridin-2-yl)piperazin-1-yl)phenyl)ethanone (34h)}

Yield: 99\%; light brown solid; ${ }^{1} \mathrm{H}$ NMR $(300 \mathrm{MHz}$, $\left.\mathrm{CDCl}_{3}\right) \delta 2.51$ (s, $3 \mathrm{H}, \mathrm{CH}_{3}$ ), 3.54-3.59 (m, 4H, $2 \mathrm{CH}_{2}$ ), 3.70 (m, 4H), 6.63-6.68 (m, 2H, Ar-H), 6.88 (d, 2H, J 9.0 Hz, Ar-H), 7.44-7.49 (m, 1H, Ar-H), 7.87 (d, 2H, J 9.0 Hz, Ar-H), 8.19 (d, 1H, J 4.2 Hz, Ar-H); ${ }^{13} \mathrm{C}$ NMR $(75 \mathrm{MHz}$, $\left.\mathrm{CDCl}_{3}\right) \delta 26.1,44.8,45.1,46.6,47.0,107.1,107.3,113.4$, 113.7, 127.8, 130.4, 137.6, 148.0, 153.9, 159.1, 196.5 .

\section{General procedure for the preparation of chalcones 9-32}

To a solution of $p$-aminated acetophenone $\mathbf{3 5 a - 3 5 h}$ $(2 \mathrm{mmol})$, carbaldehyde 36a-36c $(4 \mathrm{mmol})$ in anhydrous ethanol $(10 \mathrm{~mL})$ at room temperature, under nitrogen atmosphere, and vigorously stirred, $\mathrm{NaOH}(30 \mathrm{mmol})$ solubilized in anhydrous ethanol $(25 \mathrm{~mL})$ was added dropwise. After the complete addition of $\mathrm{NaOH}(2 \mathrm{~h})$, the reaction was kept at room temperature for two hours. Then, it was added $50 \mathrm{~mL}$ of a saturated solution of $\mathrm{NaCl}$ and the product was extracted with tert-butanol $(4 \times 20 \mathrm{~mL})$, the organic phase was washed with saturated aqueous $\mathrm{NaCl}$ solution $(3 \times 50 \mathrm{~mL})$. The aqueous phase was retro-extracted with tert-butanol $(2 \times 20 \mathrm{~mL})$. Organic phases were dried over anhydrous $\mathrm{MgSO}_{4}$ and the solvent was removed under reduced pressure. The residue was purified by column chromatography on silica gel using hexane/ethyl acetate $(7: 3,6: 4,1: 1)$ as eluent and/or by recrystallization.

(E)-3-(Pyridin-2-yl)-1-(4-(pyrrolidin-1-yl)phenyl)prop-2-en1-one (9)

Yield: 95\%; yellow solid; mp $167{ }^{\circ} \mathrm{C}$; ${ }^{1} \mathrm{H}$ NMR (300 MHz, $\left.\mathrm{CDCl}_{3}\right) \delta$ 1.99-2.04 (m, 4H, $2 \mathrm{CH}_{2}$ ), 3.34-3.39 $\left(\mathrm{m}, 4 \mathrm{H}, 2 \mathrm{CH}_{2}\right), 6.53(\mathrm{~d}, 2 \mathrm{H}, J 8.8 \mathrm{~Hz}, \mathrm{Ar}-\mathrm{H}), 7.22-7.26$ (m, 1H, Ar-H), 7.43 (d, 1H, J 7.7 Hz, Ar-H), 7.67-7.74 (m, 2H, Ar-H and CH), 8.05 (d, 2H, J $8.9 \mathrm{~Hz}, \mathrm{Ar}-\mathrm{H}), 8.15$ (d, 1H, J $15.2 \mathrm{~Hz}, \mathrm{CH}), 8.65$ (d, 1H, J $3.9 \mathrm{~Hz}, \mathrm{Ar}-\mathrm{H})$;
${ }^{13} \mathrm{C}$ NMR $\left(75 \mathrm{MHz}, \mathrm{CDCl}_{3}\right) \delta 25.4,47.6,110.9,123.9$, 125.2, 125.3, 126.1, 131.3, 136.9, 140.3, 149.4, 151.2, 153.8, 187.5; HRMS (Fourier transform mass spectrometry $($ FTMS $)+$ pESI $m / z$, calcd. for $\mathrm{C}_{18} \mathrm{H}_{18} \mathrm{~N}_{2} \mathrm{O}[\mathrm{M}]^{+}: 279.1497$, found: 279.1516 .

(E)-3-(Pyridin-3-yl)-1-(4-(pyrrolidin-1-yl)phenyl)prop-2-en1-one (10)

Yield: $85 \%$; yellow solid; mp $185{ }^{\circ} \mathrm{C} ;{ }^{1} \mathrm{H}$ NMR $\left(300 \mathrm{MHz}, \mathrm{CDCl}_{3}\right) \delta$ 2.01-2.06 (m, $\left.4 \mathrm{H}, 2 \mathrm{CH}_{2}\right), 3.36-3.40$ $\left(\mathrm{m}, 4 \mathrm{H}, 2 \mathrm{CH}_{2}\right), 6.56(\mathrm{~d}, 2 \mathrm{H}, J 9.0 \mathrm{~Hz}, \mathrm{Ar}-\mathrm{H}), 7.32(\mathrm{dd}, 1 \mathrm{H}$, $J$ 4.7, 2.7 Hz, Ar-H), 7.63 (d, 1H, J 15.7 Hz, CH), 7.73 (d, $1 \mathrm{H}, J 15.7 \mathrm{~Hz}, \mathrm{CH}), 7.91$ (dl, 1H, J 8.0 Hz, Ar-H), 7.98 (d, $2 \mathrm{H}, J 9.0 \mathrm{~Hz}, \mathrm{Ar}-\mathrm{H}), 8.57$ (dd, 1H, J 4.7, $1.3 \mathrm{~Hz}, \mathrm{Ar}-\mathrm{H})$, $8.84(\mathrm{~d}, 1 \mathrm{H}, J 1.5 \mathrm{~Hz}, \mathrm{Ar}-\mathrm{H}) ;{ }^{13} \mathrm{C} \mathrm{NMR}\left(75 \mathrm{MHz}, \mathrm{CDCl}_{3}\right) \delta$ 25.4, 47.6, 111.0, 123.8, 124.3, 125.0, 131.1, 131.5, 134.7, 138.2, 149.4, 150.1, 151.2, 186.8; HRMS (FTMS + pESI) $m / z$, calcd. for $\mathrm{C}_{18} \mathrm{H}_{18} \mathrm{~N}_{2} \mathrm{O}[\mathrm{M}]^{+}: 279.1497$, found: 279.1528 .

(E)-3-(Pyridin-4-yl)-1-(4-(pyrrolidin-1-yl)phenyl)prop-2-en1-one (11)

Yield: $65 \%$; yellow solid; mp $171-173{ }^{\circ} \mathrm{C} ;{ }^{1} \mathrm{H}$ NMR (300 MHz, $\mathrm{CDCl}_{3}$ ) $\delta$ 2.03-2.07 (m, 4H, 2 $\mathrm{CH}_{2}$ ), 3.38-3.42 (m, 4H, 2CH $), 6.57$ (d, 2H, J 9.0 Hz, Ar-H), $7.46(\mathrm{~d}, 2 \mathrm{H}$, $J 9.0 \mathrm{~Hz}, \mathrm{Ar}-\mathrm{H}), 7.64$ (d, 1H, J 15.7 Hz, CH), $7.73(\mathrm{~d}, 1 \mathrm{H}$, $J 15.7 \mathrm{~Hz}, \mathrm{CH}), 7.99$ (d, 2H, J 9.0 Hz, Ar-H), 8.65 (d, 2H, $J 5.5 \mathrm{~Hz}, \mathrm{Ar}-\mathrm{H}) ;{ }^{13} \mathrm{C}$ NMR $\left(75 \mathrm{MHz}, \mathrm{CDCl}_{3}\right) \delta 25.4,47.6$, $111.0,122.0,124.9,126.5,131.2,139.0,142.9,150.5$, 151.3, 186.7; HRMS (FTMS + pESI) $\mathrm{m} / \mathrm{z}$, calcd. for $\mathrm{C}_{18} \mathrm{H}_{18} \mathrm{~N}_{2} \mathrm{O}[\mathrm{M}]^{+}:$279.1497, found: 279.1516.

(E)-1-(4-(Piperidin-1-yl)phenyl)-3-(pyridin-2-yl)prop-2-en1-one (12)

Yield: 85\%; yellow solid; mp 156-158 ${ }^{\circ} \mathrm{C}$; ${ }^{1} \mathrm{H}$ NMR $\left(300 \mathrm{MHz}, \mathrm{CDCl}_{3}\right) \delta 1.65\left(\mathrm{~s}, 6 \mathrm{H}, 3 \mathrm{CH}_{2}\right), 3.38(\mathrm{~s}, 4 \mathrm{H}$, $\left.2 \mathrm{CH}_{2}\right), 6.86(\mathrm{~d}, 2 \mathrm{H}, J 9.0 \mathrm{~Hz}, \mathrm{Ar}-\mathrm{H}), 7.23-7.27(\mathrm{~m}, 1 \mathrm{H}$, Ar-H), $7.44(\mathrm{~d}, 1 \mathrm{H}, J$ 8.0 Hz, Ar-H),7.68-7.74 (m, 2H, Ar-H and $\mathrm{CH}), 8.04$ (d, 2H,J $9.0 \mathrm{~Hz}, \mathrm{Ar}-\mathrm{H}), 8.13(\mathrm{~d}, 1 \mathrm{H}$, $J 15.2 \mathrm{~Hz}, \mathrm{CH}), 8.66$ (d, $1 \mathrm{H}, J 3.8 \mathrm{~Hz}, \mathrm{Ar}-\mathrm{H}) ;{ }^{13} \mathrm{C}$ NMR $\left(75 \mathrm{MHz}, \mathrm{CDCl}_{3}\right) \delta$ 24.4, 25.4, 48.5, 113.2, 124.0, 125.3, 125.8, 126.9, 131.1, 136.8, 140.8, 150.0, 153.7, 154.5, 187.69; HRMS (FTMS + pESI) $m / z$, calcd. for $\mathrm{C}_{19} \mathrm{H}_{20} \mathrm{~N}_{2} \mathrm{O}$ $[\mathrm{M}]^{+}:$293.1654, found: 293.1655 .

(E)-1-(4-(Piperidin-1-yl)phenyl)-3-(pyridin-3-yl)prop-2-en1-one (13)

Yield: 90\%; yellow solid; mp 179-181 ${ }^{\circ} \mathrm{C}$; ${ }^{1} \mathrm{H}$ NMR $\left(300 \mathrm{MHz}, \mathrm{CDCl}_{3}\right) \delta 1.66\left(\mathrm{~s}, 6 \mathrm{H}, 3 \mathrm{CH}_{2}\right), 3.39\left(\mathrm{~s}, 4 \mathrm{H}, 2 \mathrm{CH}_{2}\right)$, $6.88(\mathrm{~d}, 2 \mathrm{H}, J 9.0 \mathrm{~Hz}, \mathrm{Ar}-\mathrm{H}), 7.32$ (dd, $1 \mathrm{H}, J 3.6,8.0 \mathrm{~Hz}$, Ar-H), $7.61(\mathrm{~d}, 1 \mathrm{H}, J 15.7 \mathrm{~Hz}, \mathrm{CH}), 7.74(\mathrm{~d}, 1 \mathrm{H}, J 15.7 \mathrm{~Hz}$, CH), 7.92 (d, 1H, J 8.0 Hz, Ar-H), 7.96 (d, 2H, J $9.0 \mathrm{~Hz}$, 
Ar-H), 8.57 (d, 1H, J $3.6 \mathrm{~Hz}, \mathrm{Ar}-\mathrm{H}), 8.83$ (s, 1H, Ar-H); ${ }^{13} \mathrm{C} \mathrm{NMR}\left(75 \mathrm{MHz}, \mathrm{CDCl}_{3}\right) \delta 24.3,25.3,48.4,123.7,124.0$, 126.6, 130.9, 131.2, 134.5, 138.8, 149.6, 150.5, 154.4, 187.0; HRMS (FTMS + pESI) $\mathrm{m} / z$, calcd. for $\mathrm{C}_{19} \mathrm{H}_{20} \mathrm{~N}_{2} \mathrm{O}$ $[\mathrm{M}]^{+}:$293.1654, found: 293.1653 .

(E)-1-(4-(Piperidin-1-yl)phenyl)-3-(pyridin-4-yl)prop-2-en1-one (14)

Yield: 90\%; yellow solid; mp 149-150 ${ }^{\circ} \mathrm{C}$; ${ }^{1} \mathrm{H}$ NMR $\left(300 \mathrm{MHz}, \mathrm{CDCl}_{3}\right) \delta 2.03\left(\mathrm{~s}, 6 \mathrm{H}, 3 \mathrm{CH}_{2}\right), 3.38\left(\mathrm{~s}, 4 \mathrm{H}, 2 \mathrm{CH}_{2}\right)$, 6.55 (d, 2H, J $8.0 \mathrm{~Hz}, \mathrm{Ar}-\mathrm{H}), 7.46$ (s, 2H, J $15.8 \mathrm{~Hz}, \mathrm{CH}$ ), $7.62(\mathrm{~d}, 1 \mathrm{H}, J 15.8 \mathrm{~Hz}, \mathrm{CH}), 7.72(\mathrm{~d}, 1 \mathrm{H}, J 15.8 \mathrm{~Hz}, \mathrm{CH})$, 7.97 (d, 2H, J $8.0 \mathrm{~Hz}, \mathrm{Ar}-\mathrm{H}), 8.63$ (s, 2H, Ar-H); ${ }^{13} \mathrm{C}$ NMR $\left(75 \mathrm{MHz}, \mathrm{CDCl}_{3}\right) \delta 24.3,25.3,48.4,113.2,122.6,126.3$, 127.8, 131.1, 138.5, 145.0, 148.3, 154.5, 186.4; HRMS (FTMS + pESI) $m / z$, calcd. for $\mathrm{C}_{19} \mathrm{H}_{20} \mathrm{~N}_{2} \mathrm{O}[\mathrm{M}]^{+}: 293.1654$, found: 293.1740 .

(E)-1-(4-Morpholinophenyl)-3-(pyridin-2-yl)prop-2-en1-one (15)

Yield: $81 \%$; yellow solid; mp $138-140{ }^{\circ} \mathrm{C}$; ${ }^{1} \mathrm{H}$ NMR (300 MHz, $\left.\mathrm{CDCl}_{3}\right) \delta 3.30-3.33\left(\mathrm{~m}, 4 \mathrm{H}, 2 \mathrm{CH}_{2}\right.$ ), 3.82-3.85 $\left(\mathrm{m}, 4 \mathrm{H}, 2 \mathrm{CH}_{2}\right), 6.88(\mathrm{~d}, J 9.0 \mathrm{~Hz}, 2 \mathrm{H}, \mathrm{Ar}-\mathrm{H}), 7.23-7.28$ (m, 1H, Ar-H), 7.44 (d, J 7.7 Hz, 1H, Ar-H), $7.72(\mathrm{~m}, 2 \mathrm{H}$, Ar-H and $\mathrm{CH}), 8.06$ (d, J 9.0 Hz, 2H, Ar-H), $8.13(\mathrm{~d}, 1 \mathrm{H}$, $J 15.2 \mathrm{~Hz}, \mathrm{CH}), 8.66$ (d, $1 \mathrm{H}, J 3.9 \mathrm{~Hz}, \mathrm{Ar}-\mathrm{H}) ;{ }^{13} \mathrm{C}$ NMR $\left(75 \mathrm{MHz}, \mathrm{CDCl}_{3}\right) \delta 47.4,66.5,113.3,124.1,125.3,125.7$, 128.5, 130.9, 136.9, 141.2, 150.0, 153.6, 154.3, 188.0; HRMS (FTMS + pESI) $m / z$, calcd. for $\mathrm{C}_{18} \mathrm{H}_{18} \mathrm{~N}_{2} \mathrm{O}_{2}[\mathrm{M}]^{+}$: 295.1446, found: 295.1455.

(E)-1-(4-Morpholinophenyl)-3-(pyridin-3-yl)prop-2-en1-one (16)

Yield: $91 \%$; yellow solid; mp $156-158{ }^{\circ} \mathrm{C}$; ${ }^{1} \mathrm{H}$ NMR (300 MHz, $\left.\mathrm{CDCl}_{3}\right) \delta 3.31-3.34\left(\mathrm{~m}, 4 \mathrm{H}, 2 \mathrm{CH}_{2}\right.$ ), 3.83-3.86 (m, 4H, 2CH $), 6.90(\mathrm{~d}, 2 \mathrm{H}, J 9.0 \mathrm{~Hz}, \mathrm{Ar}-\mathrm{H}), 7.33$ (dd, $J$ J.4, $4.5 \mathrm{~Hz}, 1 \mathrm{H}, \mathrm{Ar}-\mathrm{H}), 7.60(\mathrm{~d}, 1 \mathrm{H}, J 15.7 \mathrm{~Hz}, \mathrm{CH}), 7.75$ (d, 1H, J $15.7 \mathrm{~Hz}, \mathrm{CH}), 7.91$ (d, 1H, J $9.0 \mathrm{~Hz}, \mathrm{Ar}-\mathrm{H}), 7.99$ (d, $2 \mathrm{H}, J 9.0 \mathrm{~Hz}, \mathrm{Ar}-\mathrm{H}), 8.59$ (s, 1H, Ar-H), 8.84 (s, 1H, Ar-H); ${ }^{13} \mathrm{C}$ NMR (75 MHz, $\left.\mathrm{CDCl}_{3}\right) \delta 47.4,66.5,113.4$, 123.7, 123.9, 128.3, 130.8, 134.6, 135.0, 139.4, 149.7, 150.6, 154.4, 187.4; HRMS (FTMS + pESI) $\mathrm{m} / \mathrm{z}$, calcd. for $\mathrm{C}_{18} \mathrm{H}_{18} \mathrm{~N}_{2} \mathrm{O}_{2}[\mathrm{M}]^{+}:$295.1446, found: 295.1462.

(E)-1-(4-Morpholinophenyl)-3-(pyridin-4-yl)prop-2-en1-one (17)

Yield: 55\%; yellow solid; mp $122-124{ }^{\circ} \mathrm{C}$; ${ }^{1} \mathrm{H}$ NMR (300 MHz, $\left.\mathrm{CDCl}_{3}\right) \delta 3.32-3.35\left(\mathrm{~m}, 4 \mathrm{H}, 2 \mathrm{CH}_{2}\right.$ ), 3.83-3.87 $\left(\mathrm{m}, 4 \mathrm{H}, 2 \mathrm{CH}_{2}\right), 6.90(\mathrm{~d}, 2 \mathrm{H}, J 9.0 \mathrm{~Hz}, \mathrm{Ar}-\mathrm{H}), 7.45(\mathrm{dd}, 2 \mathrm{H}$, $J$ 4.5, 1.6 Hz, Ar-H), 7.63 (d, 1H, J 15.8 Hz, CH), 7.69 (d, 1H, J $15.8 \mathrm{~Hz}, \mathrm{CH}), 7.99$ (d, 2H, J 9.0 Hz, Ar-H), 8.65 (dd, 2H, J 4.5, $1.5 \mathrm{~Hz}, \mathrm{Ar}-\mathrm{H}) ;{ }^{13} \mathrm{C}$ NMR (75 MHz, $\mathrm{CDCl}_{3}$ ) $\delta$ 47.3, 66.5, 113.3, 122.0, 126.1, 128.0, 130.8, 140.0, 142.6, 150.5, 154.4, 187.2; HRMS (FTMS + pESI) $\mathrm{m} / z$, calcd. for $\mathrm{C}_{18} \mathrm{H}_{18} \mathrm{~N}_{2} \mathrm{O}_{2}[\mathrm{M}]^{+}:$295.1446, found: 295.1610.

(E)-3-(Pyridin-2-yl)-1-(4-thiomorpholinophenyl)prop-2-en1-one (18)

Yield: 90\%; yellow solid; mp $154-156{ }^{\circ} \mathrm{C}$; ${ }^{1} \mathrm{H}$ NMR $\left(300 \mathrm{MHz}, \mathrm{CDCl}_{3}\right) \delta 2.67-2.71\left(\mathrm{~m}, 4 \mathrm{H}, 2 \mathrm{CH}_{2}\right), 3.78-3.81$ (m, 4H, 2CH $\mathrm{CH}_{2}, 6.83$ (d, 2H, J 9.0 Hz, Ar-H), 7.25-7.28 (m, $1 \mathrm{H}, \mathrm{Ar}-\mathrm{H}), 7.44(\mathrm{~d}, 1 \mathrm{H}, J 7.7 \mathrm{~Hz}, \mathrm{Ar}-\mathrm{H}), 7.68-7.75(\mathrm{~m}, 2 \mathrm{H}$, Ar-H and CH), 8.05 (d, 2H, J 9.0 Hz, Ar-H), 8.11 (d, 1H, $J 15.2 \mathrm{~Hz}, \mathrm{CH}), 8.66(\mathrm{~d}, 1 \mathrm{H}, J 3.8 \mathrm{~Hz}, \mathrm{Ar}-\mathrm{H}) ;{ }^{13} \mathrm{C}$ NMR $\left(75 \mathrm{MHz}, \mathrm{CDCl}_{3}\right) \delta 25.9,50.3,113.7,124.1,125.3,125.6$, 127.7, 131.3, 136.8, 141.2, 150.1, 153.1, 153.6, 187.8; HRMS (FTMS + pESI) $m / z$, calcd. for $\mathrm{C}_{18} \mathrm{H}_{18} \mathrm{~N}_{2} \mathrm{OS}[\mathrm{M}]^{+}$: 311.1218, found: 311.1219 .

(E)-3-(Pyridin-3-yl)-1-(4-thiomorpholinophenyl)prop-2-en1-one (19)

Yield: 91\%; yellow solid; mp 120-122 ${ }^{\circ} \mathrm{C}$; ${ }^{1} \mathrm{H}$ NMR (300 MHz, $\left.\mathrm{CDCl}_{3}\right) \delta 2.68-2.71\left(\mathrm{~m}, 4 \mathrm{H}, 2 \mathrm{CH}_{2}\right), 3.79-3.83$ (m, 4H, 2 $\left.\mathrm{CH}_{2}\right), 6.85(\mathrm{~d}, 2 \mathrm{H}, J 8.7 \mathrm{~Hz}, \mathrm{Ar}-\mathrm{H}), 7.31-7.35$ (m, 1H, Ar-H), 7.60 (d, 1H, J $15.6 \mathrm{~Hz}, \mathrm{CH}), 7.75(\mathrm{~d}, 1 \mathrm{H}$, $J 15.6 \mathrm{~Hz}, \mathrm{CH}), 7.91$ (d, 1H, J 6.0 Hz, Ar-H), 7.97 (d, 2H, $J 9.0 \mathrm{~Hz}, \mathrm{Ar}-\mathrm{H}), 8.59$ (d, 1H, J 3.7 Hz, Ar-H), 8.84 (s, 1H, $\mathrm{Ar}-\mathrm{H}) ;{ }^{13} \mathrm{C}$ NMR $\left(75 \mathrm{MHz}, \mathrm{CDCl}_{3}\right) \delta 25.8,50.3,113.5$, 113.7, 123.7, 123.8, 127.3, 131.1, 134.6, 139.3, 149.7, 150.7, 153.0, 187.1; HRMS (FTMS + pESI) $\mathrm{m} / \mathrm{z}$, calcd. for $\mathrm{C}_{18} \mathrm{H}_{18} \mathrm{~N}_{2} \mathrm{OS}[\mathrm{M}]^{+}: 311.1218$, found: 311.1216 .

(E)-3-(Pyridin-4-yl)-1-(4-thiomorpholinophenyl)prop-2-en1-one (20)

Yield: 55\%; yellow solid; mp $142-144{ }^{\circ} \mathrm{C}$; ${ }^{1} \mathrm{H}$ NMR $\left(300 \mathrm{MHz}, \mathrm{CDCl}_{3}\right) \delta 2.68-2.71\left(\mathrm{~m}, 4 \mathrm{H}, 2 \mathrm{CH}_{2}\right), 3.80-3.84$ (m, $\left.4 \mathrm{H}, 2 \mathrm{CH}_{2}\right), 6.85(\mathrm{~d}, 2 \mathrm{H}, J 9.0 \mathrm{~Hz}, \mathrm{Ar}-\mathrm{H}), 7.45(\mathrm{~d}, 2 \mathrm{H}$, $J$ 6.0 Hz, Ar-H), 7.60-7.71 (m, 2H, 2CH), $7.97(\mathrm{~d}, 2 \mathrm{H}$, $J 9.0 \mathrm{~Hz}, \mathrm{Ar}-\mathrm{H}), 8.65$ (d, 2H, J 6.0 Hz, Ar-H); ${ }^{13} \mathrm{C}$ NMR $\left(75 \mathrm{MHz}, \mathrm{CDCl}_{3}\right) \delta 25.8,50.2,113.6,122.0,126.1$, 127.1, 131.2, 139.8, 142.6, 150.5, 153.1, 187.0; HRMS (FTMS + pESI) $m / z$, calcd. for $\mathrm{C}_{18} \mathrm{H}_{18} \mathrm{~N}_{2} \mathrm{OS}[\mathrm{M}]^{+}: 311.1218$, found: 311.1222 .

(E)-1-(4-(4-Methylpiperazin-1-yl)phenyl)-3-(pyridin-2-yl) prop-2-en-1-one (21)

Yield: 82\%; yellow solid; mp 143-145 ${ }^{\circ} \mathrm{C}$; ${ }^{1} \mathrm{H}$ NMR (300 MHz, $\left.\mathrm{CDCl}_{3}\right) \delta 2.34\left(\mathrm{~s}, 3 \mathrm{H}, \mathrm{CH}_{3}\right), 2.53-2.56(\mathrm{~m}, 4 \mathrm{H}$, $\left.2 \mathrm{CH}_{2}\right), 3.37-3.40\left(\mathrm{~m}, 4 \mathrm{H}, 2 \mathrm{CH}_{2}\right), 6.88(\mathrm{~d}, 2 \mathrm{H}, J 9.0 \mathrm{~Hz}$, Ar-H), 7.22-7.27 (m, 1H, Ar-H), $7.43(\mathrm{~d}, 1 \mathrm{H}, J 7.7 \mathrm{~Hz}$, Ar-H), 7.67-7.76 (m, 2H, Ar-H and CH), 8.05 (d, 2H, $J 8.8 \mathrm{~Hz}, \mathrm{Ar}-\mathrm{H}), 8.11(\mathrm{~d}, 1 \mathrm{H}, J 15.2 \mathrm{~Hz}, \mathrm{CH}), 8.62(\mathrm{~d}, 1 \mathrm{H}$, 
$J$ J.0 Hz, Ar-H); ${ }^{13} \mathrm{C}$ NMR (75 MHz, $\left.\mathrm{CDCl}_{3}\right) \delta 46.0,47.0$, 54.6, 113.5, 124.1, 125.3, 125.6, 128.0, 131.0, 136.8, 141.2, 150.1, 153.6, 154.1, 187.9; HRMS (FTMS + pESI) $\mathrm{m} / \mathrm{z}$, calcd. for $\mathrm{C}_{19} \mathrm{H}_{21} \mathrm{~N}_{3} \mathrm{O}[\mathrm{M}]^{+}: 308.1763$, found: 308.1769 .

(E)-1-(4-(4-Methylpiperazin-1-yl)phenyl)-3-(pyridin-3-yl) prop-2-en-1-one (22)

Yield: 88\%; yellow solid; mp $143{ }^{\circ} \mathrm{C}$; ${ }^{1} \mathrm{H}$ NMR $\left(300 \mathrm{MHz}, \mathrm{CDCl}_{3}\right) \delta 2.33\left(\mathrm{~s}, 3 \mathrm{H}, \mathrm{CH}_{3}\right), 2.50-2.58(\mathrm{~m}, 4 \mathrm{H}$, $\left.2 \mathrm{CH}_{2}\right), 3.34-3.43\left(\mathrm{~m}, 4 \mathrm{H}, 2 \mathrm{CH}_{2}\right), 6.90(\mathrm{~d}, 2 \mathrm{H}, J 9.0 \mathrm{~Hz}$, Ar-H), 7.32 (dd, 1H, J 8.0, 4.8 Hz, Ar-H), $7.60(\mathrm{~d}, 1 \mathrm{H}$, $J 15.7 \mathrm{~Hz}, \mathrm{CH}), 7.74$ (d, 1H, J 15.7 Hz, CH), 7.89-7.93 (m, 1H, Ar-H), 7.98 (d, 2H, J $9.0 \mathrm{~Hz}, \mathrm{Ar}-\mathrm{H}), 8.58(\mathrm{dd}, 1 \mathrm{H}$, $J$ 4.8, $1.6 \mathrm{~Hz}, \mathrm{Ar}-\mathrm{H}), 8.83$ (d, $1 \mathrm{H}, \mathrm{J} 2.0 \mathrm{~Hz}, \mathrm{Ar}-\mathrm{H}) ;{ }^{13} \mathrm{C}$ NMR $\left(75 \mathrm{MHz}, \mathrm{CDCl}_{3}\right) \delta 46.1,47.1,54.6,113.5,123.5,123.9$, 127.7, 130.8, 134.7, 134.9, 148.7, 149.6, 150.5, 154.2, 187.3; HRMS (FTMS + pESI) $m / z$, calcd. for $\mathrm{C}_{19} \mathrm{H}_{21} \mathrm{~N}_{3} \mathrm{O}$ $[\mathrm{M}]^{+}:$308.1763, found: 308.1758 .

(E)-1-(4-(4-Methylpiperazin-1-yl)phenyl)-3-(pyridin-4-yl) prop-2-en-1-one (23)

Yield: 48\%; yellow solid; mp $97{ }^{\circ} \mathrm{C}$; ${ }^{1} \mathrm{H}$ NMR $\left(300 \mathrm{MHz}, \mathrm{CDCl}_{3}\right) \delta 2.33\left(\mathrm{~s}, 3 \mathrm{H}, \mathrm{CH}_{3}\right), 2.53-2.56(\mathrm{~m}, 4 \mathrm{H}$, $\left.2 \mathrm{CH}_{2}\right), 3.37-3.40\left(\mathrm{~m}, 4 \mathrm{H}, 2 \mathrm{CH}_{2}\right), 6.88(\mathrm{~d}, J 9.0 \mathrm{~Hz}, 2 \mathrm{H}$, Ar-H), 7.44 (d, J 4.69 Hz, 2H, Ar-H), 7.61 (d, J $15.8 \mathrm{~Hz}$, $1 \mathrm{H}, \mathrm{CH}), 7.68(\mathrm{~d}, 1 \mathrm{H}, J 15.7 \mathrm{~Hz}, \mathrm{CH}), 7.96(\mathrm{~d}, 2 \mathrm{H}, J 9.0 \mathrm{~Hz}$, $\mathrm{Ar}-\mathrm{H}), 8.63$ (s, $2 \mathrm{H}, \mathrm{Ar}-\mathrm{H}) ;{ }^{13} \mathrm{C}$ NMR $\left(75 \mathrm{MHz}, \mathrm{CDCl}_{3}\right.$ ) $\delta$ 26.0, 46.9, 54.5, 113.5, 121.3, 122.0, 126.1, 127.4, 130.9, 139.8, 142.6, 150.4, 154.3, 187.1; HRMS (FTMS + pESI) $\mathrm{m} / z$, calcd. for $\mathrm{C}_{19} \mathrm{H}_{21} \mathrm{~N}_{3} \mathrm{O}[\mathrm{M}]^{+}: 308.1763$, found: 308.1759 .

(E)-1-(4-(4-Ethylpiperazin-1-yl)phenyl)-3-(pyridin-2-yl)prop2-en-1-one (24)

Yield: 94\%; yellow solid; mp $150{ }^{\circ} \mathrm{C}$; ${ }^{1} \mathrm{H}$ NMR $\left(300 \mathrm{MHz}, \mathrm{CDCl}_{3}\right) \delta 1.13\left(\mathrm{t}, 3 \mathrm{H}, J 6.0 \mathrm{~Hz}, \mathrm{CH}_{3}\right), 2.48$ (q, $\left.2 \mathrm{H}, J 6.0 \mathrm{~Hz}, \mathrm{CH}_{2}\right), 2.51-2.61\left(\mathrm{~m}, 4 \mathrm{H}, 2 \mathrm{CH}_{2}\right), 3.40-3.43$ $\left(\mathrm{m}, 4 \mathrm{H}, 2 \mathrm{CH}_{2}\right), 6.90(\mathrm{~d}, 2 \mathrm{H}, J 9.0 \mathrm{~Hz}, \mathrm{Ar}-\mathrm{H}), 7.27-7.29(\mathrm{~m}$, $1 \mathrm{H}, \mathrm{Ar}-\mathrm{H}), 7.45$ (d, 1H, J 7.7 Hz, Ar-H), 7.69-7.77 (m, 2H, Ar-H and CH), 8.06 (d, 2H, J 9.0 Hz, Ar-H), $8.13(\mathrm{~d}, 1 \mathrm{H}$, $J 15.2 \mathrm{~Hz}, \mathrm{CH}), 8.67$ (d, $1 \mathrm{H}, J 4.0 \mathrm{~Hz}, \mathrm{Ar}-\mathrm{H}) ;{ }^{13} \mathrm{C}$ NMR $\left(75 \mathrm{MHz}, \mathrm{CDCl}_{3}\right) \delta 12.0,47.2,52.3,52.5,113.3,124.1$, 125.3, 127.9, 131.0, 136.8, 141.1, 150.1, 153.6, 154.2, 187.9; HRMS (FTMS + pESI) $m / z$, calcd. for $\mathrm{C}_{20} \mathrm{H}_{23} \mathrm{~N}_{3} \mathrm{O}$ $[\mathrm{M}]^{+}:$322.1919, found: 322.1916 .

(E)-1-(4-(4-Ethylpiperazin-1-yl)phenyl)-3-(pyridin-3-yl)prop2-en-1-one (25)

Yield: 94\%; yellow solid; mp 171-173 ${ }^{\circ} \mathrm{C}$; ${ }^{1} \mathrm{H}$ NMR $\left(300 \mathrm{MHz}, \mathrm{CDCl}_{3}\right) \delta 1.17\left(\mathrm{t}, 3 \mathrm{H}, J 6.0 \mathrm{~Hz}, \mathrm{CH}_{3}\right), 2.60$ (q, $\left.2 \mathrm{H}, J 6.0 \mathrm{~Hz}, \mathrm{CH}_{2}\right), 2.53-2.67\left(\mathrm{~m}, 4 \mathrm{H}, 2 \mathrm{CH}_{2}\right), 3.36-3.54$ (m, 4H, 2CH $\mathrm{CH}_{2}, 6.91$ (d, 2H, J 9.0 Hz, Ar-H), 7.29 (dd, 1H,
J 8.0, 3.0 Hz, Ar-H), 7.60 (d, 1H, J $15.7 \mathrm{~Hz}, \mathrm{CH}), 7.75$ (d, $1 \mathrm{H}, J 15.8 \mathrm{~Hz}, \mathrm{CH}), 7.92$ (d, 2H, J 8.0 Hz, Ar-H), 7.98 (d, $1 \mathrm{H}, J 9.0 \mathrm{~Hz}, \mathrm{Ar}-\mathrm{H}), 8.59$ (dd, $1 \mathrm{H}, J$ 4.8, $1.4 \mathrm{~Hz}, \mathrm{Ar}-\mathrm{H}$ ), $8.84(\mathrm{~d}, 1 \mathrm{H}, J 1.7 \mathrm{~Hz}, \mathrm{Ar}-\mathrm{H}) ;{ }^{13} \mathrm{C}$ NMR $\left(75 \mathrm{MHz}, \mathrm{CDCl}_{3}\right)$ $\delta 11.7,46.8,47.0,52.3,113.4,113.5,123.7,123.9,127.8$, $130.8,134.5,139.3,149.8,150.7,154.1,187.3$; HRMS (FTMS + pESI) $m / z$, calcd. for $\mathrm{C}_{20} \mathrm{H}_{23} \mathrm{~N}_{3} \mathrm{O}[\mathrm{M}]^{+}: 322.1919$, found: 322.1916 .

(E)-1-(4-(4-Ethylpiperazin-1-yl)phenyl)-3-(pyridin-4-yl)prop2-en-1-one (26)

Yield: 59\%; yellow solid; mp 144-146 ${ }^{\circ} \mathrm{C}$; ${ }^{1} \mathrm{H}$ NMR $\left(300 \mathrm{MHz}, \mathrm{CDCl}_{3}\right) \delta 1.11\left(\mathrm{t}, 3 \mathrm{H}, J 6.0 \mathrm{~Hz}, \mathrm{CH}_{3}\right), 2.47$ (q, $\left.2 \mathrm{H}, J 6.0 \mathrm{~Hz}, \mathrm{CH}_{2}\right), 2.56-2.59\left(\mathrm{~m}, 4 \mathrm{H}, 2 \mathrm{CH}_{2}\right), 3.39-3.42$ (m, 4H, 2CH $), 6.89$ (d, 2H, J9.0 Hz, Ar-H), 7.44 (dd, 2H, $J$ 4.5, $1.6 \mathrm{~Hz}, \mathrm{Ar}-\mathrm{H}), 7.63(\mathrm{~d}, 1 \mathrm{H}, J 15.7 \mathrm{~Hz}, \mathrm{CH}), 7.68$ (d, 1H, J $15.7 \mathrm{~Hz}, \mathrm{CH}), 7.96$ (d, 2H, J 9.0 Hz, Ar-H), 8.63 (dd, $2 \mathrm{H}, J$ 4.5, $1.6 \mathrm{~Hz}, \mathrm{Ar}-\mathrm{H}) ;{ }^{13} \mathrm{C}$ NMR $\left(75 \mathrm{MHz}, \mathrm{CDCl}_{3}\right.$ ) $\delta 11.9,47.1,52.3,54.4,113.4,120.0,122.0,123.2,129.5$, 139.8, 142.6, 149.9, 154.3, 187.1; HRMS (FTMS + pESI) $\mathrm{m} / z$, calcd. for $\mathrm{C}_{20} \mathrm{H}_{23} \mathrm{~N}_{3} \mathrm{O}[\mathrm{M}]^{+}: 322.1919$, found: 322.1901 .

(E)-3-(Pyridin-2-yl)-1-(4-(4-(pyrimidin-2-yl)piperazin-1-yl) phenyl)prop-2-en-1-one (27)

Yield: 87\%; yellow solid; mp 180-182 ${ }^{\circ} \mathrm{C}$; ${ }^{1} \mathrm{H}$ NMR (300 MHz, $\left.\mathrm{CDCl}_{3}\right) \delta 3.46-3.50\left(\mathrm{~m}, 4 \mathrm{H}, 2 \mathrm{CH}_{2}\right.$ ), 3.96-4.00 $\left(\mathrm{m}, 4 \mathrm{H}, 2 \mathrm{CH}_{2}\right), 6.53(\mathrm{t}, 1 \mathrm{H}, J 4.7 \mathrm{~Hz}, \mathrm{Ar}-\mathrm{H}), 6.93(\mathrm{~d}$, 2H, J 8.9 Hz, Ar-H), 7.23-7.26 (m, 1H, Ar-H), 7.45 (d, $1 \mathrm{H}, J 7.7 \mathrm{~Hz}, \mathrm{Ar}-\mathrm{H}), 7.69-7.76(\mathrm{~m}, 2 \mathrm{H}, \mathrm{Ar}-\mathrm{H}$ and $\mathrm{CH})$, 8.06-8.15 (m, 3H, Ar-H and CH), $8.33(\mathrm{~d}, 2 \mathrm{H}, J 4.7 \mathrm{~Hz}$, Ar-H), 8.67 (d, 1H, J 4.1 Hz, Ar-H); ${ }^{13} \mathrm{C}$ NMR $(75 \mathrm{MHz}$, $\left.\mathrm{CDCl}_{3}\right) \delta$ 43.2, 47.0, 110.4, 113.5, 124.2, 125.5, 125.8, 128.1, 131.1, 140.9, 149.8, 153.4, 154.1, 157.8, 165.8, 187.9; HRMS (FTMS + pESI) $\mathrm{m} / z$, calcd. for $\mathrm{C}_{22} \mathrm{H}_{21} \mathrm{~N}_{5} \mathrm{O}$ $[\mathrm{M}]^{+}:$372.1824, found: 372.1844 .

(E)-3-(Pyridin-3-yl)-1-(4-(4-(pyrimidin-2-yl)piperazin-1-yl) phenyl)prop-2-en-1-one (28)

Yield: $36 \%$; yellow solid; mp 208-210 ${ }^{\circ} \mathrm{C}$; ${ }^{1} \mathrm{H}$ NMR (300 MHz, $\mathrm{CDCl}_{3}$ ) $\delta$ 3.42-3.44 (m, 4H, $2 \mathrm{CH}_{2}$ ), 3.93-3.96 (m, 4H, 2CH $), 6.49$ (t, 1H, J 4.7 Hz, Ar-H), 6.89 (d, 2H, $J$ 9.0 Hz, Ar-H), 7.29 (dd, 1H, J 7.9, 4.9 Hz, Ar-H), 7.56 (d, 1H, J15.7 Hz, CH), 7.71 (d, 1H, J $15.7 \mathrm{~Hz}, \mathrm{CH}), 7.88$ (dd, $1 \mathrm{H}, J 6.1,1.9 \mathrm{~Hz}, \mathrm{Ar}-\mathrm{H}), 7.96$ (d, 2H, J $9.0 \mathrm{~Hz}, \mathrm{Ar}-\mathrm{H}$ ), 8.29 (d, 2H, J 4.7 Hz, Ar-H), 8.55 (d, 1H, J 3.7 Hz, Ar-H), 8.80 (s, $1 \mathrm{H}, \mathrm{Ar}-\mathrm{H}) ;{ }^{13} \mathrm{C}$ NMR $\left(75 \mathrm{MHz}, \mathrm{CDCl}_{3}\right) \delta 43.2$, 47.0, 110.4, 113.3, 113.5, 123.7, 123.9, 127.9, 130.8, 131.2, 134.5, 139.3, 149.8, 150.7, 154.1, 157.8, 187.0; HRMS (FTMS + pESI) $\mathrm{m} / z$, calcd. for $\mathrm{C}_{22} \mathrm{H}_{21} \mathrm{~N}_{5} \mathrm{O}[\mathrm{M}]^{+}: 372.1824$, found: 372.1834 . 
(E)-3-(Pyridin-4-yl)-1-(4-(4-(pyrimidin-2-yl)piperazin-1-yl) phenyl)prop-2-en-1-one (29)

Yield: 94\%; yellow solid; mp 207-208 ${ }^{\circ} \mathrm{C}$; ${ }^{1} \mathrm{H}$ NMR $\left(300 \mathrm{MHz}, \mathrm{CDCl}_{3}\right) \delta 3.48-3.52\left(\mathrm{~m}, 4 \mathrm{H}, 2 \mathrm{CH}_{2}\right.$ ), 3.97-4.01 $\left(\mathrm{m}, 4 \mathrm{H}, 2 \mathrm{CH}_{2}\right), 6.54(\mathrm{~m}, 1 \mathrm{H}, J 4.7 \mathrm{~Hz}, \mathrm{Ar}-\mathrm{H}), 6.93(\mathrm{~d}, 2 \mathrm{H}$, $J 9.0 \mathrm{~Hz}, \mathrm{Ar}-\mathrm{H}), 7.46(\mathrm{dd}, 2 \mathrm{H}, J$ 6.0, $1.5 \mathrm{~Hz}, \mathrm{Ar}-\mathrm{H}), 7.64(\mathrm{~d}$, $1 \mathrm{H}, J 15.7 \mathrm{~Hz}, \mathrm{CH}), 7.70(\mathrm{~d}, 1 \mathrm{H}, J 15.7 \mathrm{~Hz}, \mathrm{CH}), 8.00$ (d, $2 \mathrm{H}, J 9.0 \mathrm{~Hz}, \mathrm{Ar}-\mathrm{H}$ ), 8.33 (d, 2H, J 4.7 Hz, Ar-H), 8.65 (d, $2 \mathrm{H}, J 5.6 \mathrm{~Hz}, \mathrm{Ar}-\mathrm{H}) ;{ }^{13} \mathrm{C} \mathrm{NMR}\left(75 \mathrm{MHz}, \mathrm{CDCl}_{3}\right) \delta 43.2$, $46.9,110.4,113.4,122.0,126.1,127.6,130.9,139.9,142.6$, 150.5, 154.2, 157.8, 161.5, 187.2; HRMS (FTMS + pESI) $\mathrm{m} / z$, calcd. for $\mathrm{C}_{22} \mathrm{H}_{21} \mathrm{~N}_{5} \mathrm{O}[\mathrm{M}]^{+}: 372.1824$, found: 372.1826 .

(E)-3-(Pyridin-2-yl)-1-(4-(4-(pyridin-2-yl)piperazin-1-yl) phenyl)prop-2-en-1-one (30)

Yield: 50\%; yellow solid; mp 152-154 ${ }^{\circ} \mathrm{C} ;{ }^{1} \mathrm{H}$ NMR (300 MHz, $\mathrm{CDCl}_{3}$ ) $\delta 3.51-3.55\left(\mathrm{~m}, 4 \mathrm{H}, \mathrm{CH}_{2}\right.$ ), 3.70-3.74 $\left(\mathrm{m}, 4 \mathrm{H}, \mathrm{CH}_{2}\right), 6.63-6.68(\mathrm{~m}, 2 \mathrm{H}, \mathrm{Ar}-\mathrm{H}), 6.92(\mathrm{~d}, 2 \mathrm{H}$, $J$ 9.0 Hz, Ar-H), 7.25-7.28 (m, 1H, Ar-H), $7.44(\mathrm{~d}, 1 \mathrm{H}$, $J$ 7.7 Hz, Ar-H), 7.47-7.53 (m, 1H, Ar-H), 7.68-7.76 (m, $2 \mathrm{H}, \mathrm{Ar}-\mathrm{H}$ and $\mathrm{CH}$ ), 8.08 (d, 2H, J 9.0 Hz, Ar-H), 8.13 (d, $1 \mathrm{H}, J 15.2 \mathrm{~Hz}, \mathrm{CH}), 8.20(\mathrm{dd}, 1 \mathrm{H}, J 4.2,1.1 \mathrm{~Hz}, \mathrm{Ar}-\mathrm{H})$, 8.67 (d, $1 \mathrm{H}, J 3.3 \mathrm{~Hz}, \mathrm{Ar}-\mathrm{H}) ;{ }^{13} \mathrm{C}$ NMR $\left(75 \mathrm{MHz}, \mathrm{CDCl}_{3}\right)$ $\delta 44.7,46.9,107.1,113.4,113.7,123.7,123.9,127.8$, 130.8, 131.1, 134.5, 137.6, 139.3, 148.0, 149.8, 150.7, 154.0, 159.0, 187.3; HRMS (FTMS + pESI) $\mathrm{m} / \mathrm{z}$, calcd. for $\mathrm{C}_{23} \mathrm{H}_{22} \mathrm{~N}_{4} \mathrm{O}[\mathrm{M}]^{+}:$371.1872, found: 371.1872 .

(E)-1-(4-(4-(Pyridin-2-yl)piperazin-1-yl)phenyl)-3-(pyridin3-yl)prop-2-en-1-one (31)

Yield: 73\%; yellow solid; mp 192-194 ${ }^{\circ} \mathrm{C}$; ${ }^{1} \mathrm{H}$ NMR (300 MHz, $\left.\mathrm{CDCl}_{3}\right) \delta 3.52-3.55\left(\mathrm{~m}, 4 \mathrm{H}, 2 \mathrm{CH}_{2}\right.$ ), 3.71-3.74 (m, 4H, 2 $\left.\mathrm{CH}_{2}\right), 6.65-6.68(\mathrm{~m}, 2 \mathrm{H}, \mathrm{Ar}-\mathrm{H}), 6.93(\mathrm{~d}, 2 \mathrm{H}$, $J 9.0 \mathrm{~Hz}, \mathrm{Ar}-\mathrm{H}), 7.31-7.35$ (m, 1H, Ar-H), 7.48-7.53 (m, 1H, Ar-H), 7.61 (d, $1 \mathrm{H}, J 15.9 \mathrm{~Hz}, \mathrm{CH}), 7.75(\mathrm{~d}, 1 \mathrm{H}, J 15.9 \mathrm{~Hz}$, CH), $7.92(\mathrm{~d}, 1 \mathrm{H}, J 9.0 \mathrm{~Hz}, \mathrm{Ar}-\mathrm{H}), 8.01(\mathrm{~d}, 2 \mathrm{H}, J 9.0 \mathrm{~Hz}$, Ar-H), 8.19 (m, 1H, Ar-H), 8.59 (m, 1H, Ar-H), 8.84 (d, $1 \mathrm{H}, J 1.8 \mathrm{~Hz}, \mathrm{Ar}-\mathrm{H}) ;{ }^{13} \mathrm{C}$ NMR $\left(75 \mathrm{MHz}, \mathrm{CDCl}_{3}\right) \delta 44.7$, 76.9, 107.1, 113.4, 113.7, 123.7, 123.9, 127.8, 130.8, 131.1, $134.5,137.6,139.3,148.0,149.8,150.7,154.0,159.0$, 187.3; HRMS (FTMS + pESI) $\mathrm{m} / z$, calcd. for $\mathrm{C}_{23} \mathrm{H}_{22} \mathrm{~N}_{4} \mathrm{O}$ $[\mathrm{M}]^{+}:$371.1872, found: 371.1876 .

(E)-1-(4-(4-(Pyridin-2-yl)piperazin-1-yl)phenyl)-3-(pyridin4-yl)prop-2-en-1-one (32)

Yield: $73 \%$; yellow solid; mp 214-216 ${ }^{\circ} \mathrm{C}$; ${ }^{1} \mathrm{H}$ NMR (300 MHz, $\mathrm{CDCl}_{3}$ ) $\delta 3.53-3.57\left(\mathrm{~m}, 4 \mathrm{H}, 2 \mathrm{CH}_{2}\right.$ ), 3.72-3.75 $\left(\mathrm{m}, 4 \mathrm{H}, 2 \mathrm{CH}_{2}\right), 6.64-6.68(\mathrm{~m}, 2 \mathrm{H}, \mathrm{Ar}-\mathrm{H}), 6.94(\mathrm{~d}, 2 \mathrm{H}$, $J 9.0 \mathrm{~Hz}, \mathrm{Ar}-\mathrm{H}$ ), 7.46 (dd, 2H, J 6.0, $1.6 \mathrm{~Hz}, \mathrm{Ar}-\mathrm{H}$ ), 7.48$7.54(\mathrm{~m}, 1 \mathrm{H}, \mathrm{Ar}-\mathrm{H}), 7.64(\mathrm{~d}, 1 \mathrm{H}, J 15.7 \mathrm{~Hz}, \mathrm{CH}), 7.70(\mathrm{~d}$, $1 \mathrm{H}, J 15.7 \mathrm{~Hz}, \mathrm{CH}), 8.00$ (d, 2H, J 9.0 Hz, Ar-H), 8.19-8.23 (m, Ar-H), 8.66 (dd, 2H, J 6.0, 1.6 Hz, Ar-H); ${ }^{13} \mathrm{C}$ NMR $\left(75 \mathrm{MHz}, \mathrm{CDCl}_{3}\right) \delta 44.7,46.8,107.1,113.3,113.8,122.0$, 126.1, 127.5, 130.9, 137.7, 139.9, 142.6, 148.0, 150.5, 154.1, 159.0, 187.2; HRMS (FTMS + pESI) $\mathrm{m} / \mathrm{z}$, calcd. for $\mathrm{C}_{23} \mathrm{H}_{22} \mathrm{~N}_{4} \mathrm{O}[\mathrm{M}]^{+}: 371.1872$, found: 371.1872 .

\section{Antitubercular assay}

The strains of M. tuberculosis H37Rv (ATCC27294) were grown in the Ogawa-Kudoh medium for 10 days at $37{ }^{\circ} \mathrm{C}$. For testing, a colony was removed and cultured in Middlebrook 7H9 broth (Difco ${ }^{\circledR}$, Franklin Lakes, NJ, United States) supplemented with oleic acid, bovine serum albumin, dextrose and catalase (OADC enrichment BBL/ Becton-Dickinson $^{\circledast}$, Franklin Lakes, NJ, United States), and it was also added $0.5 \%$ glycerol as carbon source and $0.5 \%$ Tween 80 (Sigma-Aldrich ${ }^{\circledR}$, St. Louis, MO, USA) to prevent the appearance of lumps. The broth was maintained for 15 days at $37^{\circ} \mathrm{C}$. The bacterial suspensions were prepared and adjusted to the No. 1 McFarland scale. The stock solutions of tested extract, fractions, and isolated compounds were solubilized in DMSO (SigmaAldrich $^{\circledast}$, St. Louis, MO, USA) and diluted in Middlebrook $7 \mathrm{H} 9$ broth (Difco ${ }^{\circledR}$, Franklin Lakes, NJ, United States) supplemented with bovine serum and OADC enrichment (oleic albumin dextrose catalase) (BBL/Becton Dickinson ${ }^{\circledR}$, Franklin Lakes, NJ, United States). Rifampicin and isoniazid were solubilized according to the manufacturer's recommendations (Sigma-Aldrich ${ }^{\circledR}$, St. Louis, MO, USA) and used as positive control drugs. The determination of antimycobacterial activity was performed using the resazurin microtiter assay. ${ }^{26}$ Briefly, $100 \mu \mathrm{L}$ of Middlebrook $7 \mathrm{H} 9$ broth (Difco ${ }^{\circledast}$, Franklin Lakes, NJ, United States) supplemented was dispensed in each well of a sterile flat-bottom 96-well plate, then serial dilutions were made of solutions in order to obtain different concentrations of tested compounds ( 0.98 to $250 \mu \mathrm{g} \mathrm{mL}^{-1}$ ) and reference drug ( 0.004 to $1 \mu \mathrm{g} \mathrm{mL}^{-1}$ ). After dilutions, $100 \mu \mathrm{L}$ of bacterial suspension $\left(5 \times 10^{5} \mathrm{CFU} \mathrm{mL} \mathrm{mL}^{-1}\right)$ was added to each well. Plates were incubated for 7 days at $37^{\circ} \mathrm{C}$, after this period, $30 \mu \mathrm{L}$ of resazurin solution (Sigma-Aldrich ${ }^{\circledast}$, St. Louis, MO, USA) diluted in $0.01 \%$ sterile water was added to each well and the samples were incubated for $24 \mathrm{~h}$ at $37^{\circ} \mathrm{C}$. The reading was performed based on the color change and the absorbance on a microplate reader TP-Reader (Thermo Plate ${ }^{\circledR}$ ) at a wavelength of $492 \mathrm{~nm}$. Each compound was analyzed in triplicate on alternate days. The MIC was defined as the lowest concentration that results in $90 \%$ inhibition of growth of M. tuberculosis. ${ }^{27}$ Compounds with an MIC value $<100 \mu \mathrm{g} \mathrm{mL}^{-1}$ were defined as active against M. tuberculosis. Several authors ${ }^{28}$ consider MIC values of 
plant samples less than $200 \mu \mathrm{g} \mathrm{mL} \mathrm{m}^{-1}$ as being active against M. tuberculosis. However, for this to work, we suggest the following MIC values: inactive, $\geq 100 \mu \mathrm{g} \mathrm{mL}^{-1}$; moderate, $\geq 10$ and $\leq 100 \mu \mathrm{g} \mathrm{mL}^{-1}$; and high, $\leq 10 \mu \mathrm{gL}^{-1}$.

\section{Cytotoxicity assay}

Fibroblasts (NIH/3T3) obtained from Rio de Janeiro Cell Bank (Brazil) were seeded in 96-well plates $\left(1 \times 10^{4}\right.$ cells $\left.\mathrm{mL}^{-1}\right)$ and incubated with synthetic compounds at $37^{\circ} \mathrm{C}, 5 \% \mathrm{CO}_{2}$ for $48 \mathrm{~h}$ at the concentrations of $0.25-250 \mu \mathrm{g} \mathrm{mL}{ }^{-1}{ }^{29,30}$ Doxorubicin (Sigma-Aldrich $^{\circledR}$, St. Louis, MO, USA) was used as the reference drug at concentrations of $0.025-25 \mu \mathrm{g} \mathrm{mL}^{-1}$. Cell growth was estimated by the sulforhodamine B colorimetric method (SRB). ${ }^{31}$ DMSO (Vetec ${ }^{\circledR}$, Rio de Janeiro, RJ, Brazil) was used as a negative control at the concentration necessary to solubilize the highest concentration of the test samples and did not interfere with cell viability. The percentage of growth was calculated as described by Monks et al..$^{32} \mathrm{IC}_{50}$ (half maximal inhibitory concentration on fibroblast cells) was determined by nonlinear regression analysis (Microcal Origin $6.0^{\circledR 33}$ and Microsoft Office Excel $2007^{\circledR}$ ). Selectivity index (SI) was calculated according to de Medeiros et al. ${ }^{34}$

\section{Supplementary Information}

Supplementary data are available free of charge at http://jbcs.sbq.org.br as PDF file.

\section{Acknowledgments}

This study was supported by grants from FUNDECTMS, PROPP-UFMS, CAPES, and CNPq.

\section{Author Contributions}

Adriano C. M. Baroni was responsible for the conceptualization, resources, writing original draft, review and editing; Júlio Croda for the resources and antimycobacterial experiments; Renata T. Perdomo for the resources and cytotoxicity experiments; Jefferson R. S. Oliveira for the synthesis of chalcones and writing original draft; Flora M. F. Moreira for the antimycobacterial experiments; Giovana B. Gomes for the cytotoxicity experiments; Cristiane Y. K. Shiguemoto for the writing of the original draft; Amarith R. das Neves for the writing of original draft, review and editing; Sandro L. Barbosa and Palimécio G. Guerrero Jr. for the writing review and editing.

\section{References}

1. World Health Organization (WHO); Global Tuberculosis Report 2018; WHO: Geneva, 2018.

2. Brooks, G. F.; Carroll, K. C.; Butel, S. J.; Morse, S. A.; Mietzner, T. A.; Microbiologia Médica; McGraw Hill do Brasil: Rio de Janeiro, Brazil, 2009.

3. World Health Organization (WHO); Treatment of Tuberculosis: Guidelines, $4^{\text {th }}$ ed.; WHO Press: Geneva, 2010.

4. Souza, M. V. N.; Vasconcelos, T. R. A.; Quim. Nova 2005, 28, 678.

5. https://tbfacts.org/tb-drugs/, accessed in December 2019.

6. https://tbfacts.org/mdr-tb/, accessed in December 2019.

7. Zumbla, A. L.; Gillespie, S. H.; Hoelscher, M.; Philips, P. P. J.; Cole, S. T.; Abubakar, I.; McHugh, T. D.; Schito, M.; Maeurer, M.; Nunn, A. J. N.; Lancet Infect. Dis. 2014, 14, 327.

8. Brahmachari, G. In Bioactive Natural Products: Opportunities and Challenges in Medicinal Chemistry, $1^{\text {st }}$ ed.; Brahmachari, G., ed.; World Scientific Publishing: Singapore, 2011, p. 1.

9. Balunas, M. J.; Kinghorn, D. A.; Life Sci. 2005, 78, 431.

10. Zhuang, C.; Zhang, W.; Sheng, C.; Zhang, W.; Xing, C.; Miao, Z.; Chem. Rev. 2017, 117, 7762.

11. Lin, Y. M.; Zhou, Y.; Flavin, M. T.; Zhou, L. M.; Nie, W.; Chen, F. C.; Bioorg. Med. Chem. 2002, 10, 2795.

12. Patel, J. R.; Dholakia, B. Z.; Med. Chem. Res. 2012, 21, 1977.

13. Hans, R. H.; Guantai, E. M.; Carmen, L.; Smith, P. J.; Wan, B.; Franzblau, S. G.; Gut, J.; Rosenthal, P. J.; Chibale, K.; Bioorg. Med. Chem. Lett. 2010, 20, 942.

14. Macaev, F.; Boldescu, V.; Pogrebnoi, S.; Duca, G.; Med. Chem. 2014, 4, 487.

15. Chiaradia, L. D.; Mascarello, A. M.; Purificação, M.; Vernal, J.; Cordeiro, M. N. S.; Zenteno, M. E.; Villarino, A.; Nunes, R. J.; Yunes, R. A.; Terenzi, H.; Bioorg. Med. Chem. Lett. 2008, 18, 6227.

16. Tawari, N. R.; Bairwas, R.; Ray, M. K.; Rajan, M. G. R.; Degani, M. S.; Bioorg. Med. Chem. Lett. 2010, 20, 6175.

17. Gomes, M. N.; Braga, R. C.; Grzelak, E. M.; Neves, B. J.; Muratov, E.; Ma, R.; Klein, L. L.; Cho, S.; Oliveira, G. R.; Franzblau, S. G.; Andrade, C. H.; Eur. J. Med. Chem. 2017, 137, 126.

18. Anandan, R.; Jadav, S. S.; Ala, V. B.; Ahsan, M. J.; Bollikolla, H. B.; Med. Chem. Res. 2018, 27, 1690.

19. Muradás, T. C.; Abbadi, B. L.; Villela, A. D.; Macchi, F. S.; Bergo, P. F.; Freitas, T. F.; Sperotto, N. D. M.; Timmers, L. F. S. M.; Souza, O. N.; Picada, J. N.; Facchini, J.; Silva, J. B.; Albuquerque, N. C. P.; Habenschus, M. D.; Carrão, D. B.; Rocha, B. A.; Barbosa Jr., F.; Oliveira, A. R. M.; Mascarello, A.; Neuenfeldf, P.; Nunes, R. J.; Morbidoni, H. R.; Campos, M. M.; Basso, L. A.; Rodrigues-Junior, V. S.; PLoS One 2018, 13, e0202568. 
20. Wermuth, C. G.; The Practice of Medicinal Chemistry, $3^{\text {rd }}$ ed.; Academic Press: San Diego, 2008.

21. Vilchèze, C.; Jacobs Jr., W. R.; Annu. Rev. Microbiol. 2007, 61, 35.

22. Berning, S. E.; Drugs 2001, 61, 9.

23. Alcalá, L.; Ruiz-Serrano, M. J.; Turégano, C. P.; Viedma, D. G.; Díaz-Infantes, M.; Marín-Arriaza, M.; Bouza, E.; Antimicrob. Agents Chemother. 2003, 47, 416.

24. Ye, G.; Kan, W.; Yang, J.; Zeng, Z.; Liu, X.; e-Polym. 2010, 10, DOI 10.1515/epoly.2010.10.1.1533.

25. Krohn, K.; Ahmed, I.; John, M.; Synthesis 2009, 5, 779.

26. Palomino, J. C.; Martin, A.; Camacho, M.; Guerra, H.; Swings, J.; Portaels, F.; Antimicrob. Agents Chemother. 2002, 46, 2720.

27. Pavan, F. R.; Poelhsitz, G. V.; Barbosa, M. I.; Leite, S. R.; Batista, A. A.; Ellena, J.; Sato, L. S.; Franzblau, S. G.; Moreno, V.; Gambino, D.; Leite, C. Q. F.; Eur. J. Med. Chem. 2011, 46, 5099.

28. Negi, A. S.; Kumar, J. K.; Luqman, S.; Saikia, D.; Khanuja, S. P.; Med. Res. Rev. 2010, 30, 603.
29. Cassamale, T. B.; Costa, E. C.; Carvalho, D. B.; Cassemiro, N. S.; Tomazela, C. C.; Marques, M. C. S.; Ojeda, M.; Matos, M. F. C.; Albuquerque, S.; Arruda, C. C. P.; Baroni, A. C. M.; J. Braz. Chem. Soc. 2016, 27, 1217.

30. Aleixo, M. A. A.; Garcia, T. M.; Carvalho, D. B.; Viana, L. H.; Amaral, M. S.; Kassab, N. M.; Cunha, M. C.; Pereira, I. C.; Guerrero Jr., P. G.; Perdomo, R. T.; Matos, M. F. C.; Baroni, A. C. M.; J. Braz. Chem. Soc. 2018, 29, 109.

31. Skehan, P.; Storeng, R.; Scudiero, D.; Monks, A.; Mcmahon, J.; Vistica, D.; JNCI, J. Natl. Cancer Inst. 1990, 82, 1107.

32. Monks, A. D.; Scudiero, D. A.; Skehan, P.; Shoemaker, R. H.; Paull, K. D.; Vistica, D. T.; JNCI, J. Natl. Cancer Inst. 1991, $83,757$.

33. Origin, version 6.0; Microcal Software, Inc., Northampton, MA, USA, 1999.

34. de Medeiros, M. G. F.; da Silva, A. C.; Citó, A. M. G. L.; Borges, A. R.; de Lima, S. G.; Lopes, J. A. D.; Figueiredo, R. C. B. Q.; Parasitol. Int. 2011, 60, 237.

Submitted: October 11, 2019 Published online: January 30, 2020 